\title{
Wireless Sensor Network Localization Techniques
}

\author{
Guoqiang Mao, Barış Fidan and Brian D.O. Anderson
}

\begin{abstract}
Wireless sensor network localization is an important area that attracted significant research interest. This interest is expected to grow further with the proliferation of wireless sensor network applications. This paper provides an overview of the measurement techniques in sensor network localization and the one-hop localization algorithms based on these measurements. A detailed investigation on multihop connectivity-based and distance-based localization algorithms are presented. A list of open research problems in the area of distance-based sensor network localization is provided with discussion on possible approaches to them.
\end{abstract}

Index Terms-wireless sensor networks, localization, AOA, RSS, TDOA.

\section{INTRODUCTION}

W IRELESS sensor networks (WSNs) are a significant technology attracting considerable research interest. Recent advances in wireless communications and electronics have enabled the development of low-cost, low-power and multi-functional sensors that are small in size and communicate in short distances. Cheap, smart sensors, networked through wireless links and deployed in large numbers, provide unprecedented opportunities for monitoring and controlling homes, cities, and the environment. In addition, networked sensors have a broad spectrum of applications in the defence area, generating new capabilities for reconnaissance and surveillance as well as other tactical applications [1].

Self-localization capability is a highly desirable characteristic of wireless sensor networks. In environmental monitoring applications such as bush fire surveillance, water quality monitoring and precision agriculture, the measurement data are meaningless without knowing the location from where the data are obtained. Moreover, location estimation may enable a myriad of applications such as inventory management, intrusion detection, road traffic monitoring, health monitoring, reconnaissance and surveillance.

Sensor network localization algorithms estimate the locations of sensors with initially unknown location information by using knowledge of the absolute positions of a few sensors and inter-sensor measurements such as distance and bearing measurements. Sensors with known location information are called anchors and their locations can be obtained by using a global positioning system (GPS), or by installing anchors at points with known coordinates. In applications requiring a global coordinate system, these anchors will determine the location of the sensor network in the global coordinate system.

G. Mao is with the University of Sydney and National ICT Australia, Sydney. B. Fidan and B. D. O. Anderson are with the Australian National University and National ICT Australia. National ICT Australia is funded by the Australian Governments Department of Communications, Information Technology and the Arts and the Australian Research Council through the Backing Australias Ability initiative and the ICT Centre of Excellence Program.
In applications where a local coordinate system suffices (e.g., smart homes), these anchors define the local coordinate system to which all other sensors are referred. Because of constraints on the cost and size of sensors, energy consumption, implementation environment (e.g., GPS is not accessible in some environments) and the deployment of sensors (e.g., sensor nodes may be randomly scattered in the region), most sensors do not know their locations. These sensors with unknown location information are called non-anchor nodes and their coordinates will be estimated by the sensor network localization algorithm.

In this paper, we provide an overview of techniques that can be used for WSN localization. Review of wireless network localization techniques can be found in [2], [3], [4]. The focus of these references is on localization techniques in cellular network and wireless local area network (WLAN) environments and on the signal processing aspect of localization techniques. Sensor networks vary significantly from traditional cellular networks and WLAN, in that sensor nodes are assumed to be small, inexpensive, cooperative and deployed in large quantity. These features of sensor networks present unique challenges and opportunities for WSN localization. Patwari et al. described some general signal processing tools that are useful in cooperative WSN localization algorithms [5] with a focus on computing the Cramér-Rao bounds for localization using a variety of different types of measurements [5]. Our review in contrast focuses on the measurement techniques and localization algorithms in WSNs. While many techniques covered in this paper can be applied in both 2-dimension $\left(\Re^{2}\right)$ and 3-dimension $\left(\Re^{3}\right)$, we choose to focus on 2D localization problems for ease of explanation.

The rest of the paper is organized as follows. In Section II, measurement techniques in WSN localization are discussed; these include angle-of-arrival (AOA) measurements, distance related measurements and RSS profiling techniques. Distance related measurements are further classified into one-way propagation time and roundtrip propagation time measurements, the lighthouse approach to distance measurements, received signal strength (RSS)-based distance measurements and timedifference-of-arrival (TDOA) measurements. In Section III, one-hop localization techniques based on these measurements are discussed. Section IV discusses nonline-of-sight error mitigation techniques in WSN localization. Section V and Section VI focus on multihop localization techniques, in particular connectivity-based and distance-based multihop localization techniques. Section VII discusses open research problems in distance-based localization. Finally a summary is provided in Section VIII.

\section{Measurement Techniques}

Measurement techniques in WSN localization can be broadly classified into three categories: AOA measurements, 
distance related measurements and RSS profiling techniques.

\section{A. Angle-of-arrival measurements}

The angle-of-arrival measurement techniques can be further divided into two subclasses: those making use of the receiver antenna's amplitude response and those making use of the receiver antenna's phase response.

Beamforming is the name given to the use of anisotropy in the reception pattern of an antenna, and it is the basis of one category of AOA measurement techniques. The measurement unit can be of small size in comparison with the wavelength of the signals. The beam pattern of a typical anisotropic antenna is shown in Fig. 1. One can imagine that the beam of the receiver antenna is rotated electronically or mechanically, and the direction corresponding to the maximum signal strength is taken as the direction of the transmitter. Relevant parameters are the sensitivity of the receiver and the beam width. A technical problem to be faced and overcome arises when the transmitted signal has a varying signal strength. The receiver cannot differentiate the signal strength variation due to the varying amplitude of the transmitted signal and the signal strength variation caused by the anisotropy in the reception pattern. One approach to dealing with the problem is to use a second non-rotating and omnidirectional antenna at the receiver. By normalizing the signal strength received by the rotating anisotropic antenna with respect to the signal strength received by the non-rotating omnidirectional antenna, the impact of varying signal strength can be largely removed.

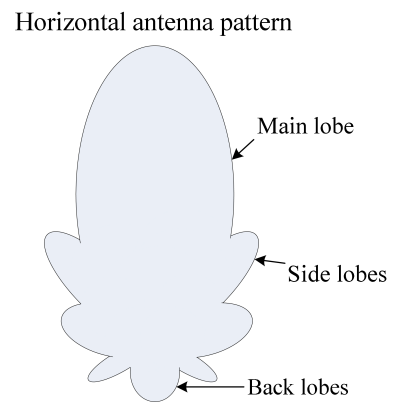

Fig. 1. An illustration of the horizontal antenna pattern of a typical anisotropic antenna.

Another widely used approach [6] to cope with the varying signal strength problem is to use a minimum of two (but typically at least four) stationary antennas with known, anisotropic antenna patterns. Overlapping of these patterns and comparing the signal strength received from each antenna at the same time yields the transmitter direction, even when the signal strength changes. Coarse tuning is performed by measuring which antenna has the strongest signal, and it is followed by fine tuning which compares amplitude responses. Because small errors in measuring the received power can lead to a large AOA measurement error, a typical measurement accuracy for four antennas is $10-15$ degrees. With six antennas, this can be improved to about 5 degrees, and 2 degrees with eight antennas [6].
The second category of measurement techniques, known as phase interferometry [7], derives the AOA measurements from the measurements of the phase differences in the arrival of a wave front. It typically requires a large receiver antenna (relative to the wavelength of the transmitter signal) or an antenna array. Fig. 2 shows an antenna array of $N$ antenna elements. The adjacent antenna elements are separated by a uniform distance $d$. The distance between a transmitter far away from the antenna array and the $i^{\text {th }}$ antenna element can be approximated by

$$
R_{i} \approx R_{0}-i d \cos \theta
$$

where $R_{0}$ is the distance between the transmitter and the $0^{\text {th }}$ antenna element and $\theta$ is the bearing of the transmitter with respect to the antenna array. The transmitter signals received by adjacent antenna elements have a phase difference of $2 \pi \frac{d \cos \theta}{\lambda}$, which allows us to obtain the bearing of the transmitter from the measurement of the phase difference. This approach works quite well for high SNR but may fail in the presence of strong co-channel interference and/or multipath signals [7].

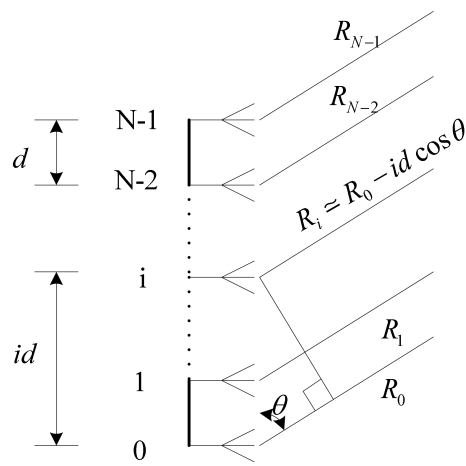

Fig. 2. An antenna array with $\mathrm{N}$ antenna elements.

The accuracy of AOA measurements is limited by the directivity of the antenna, by shadowing and by multipath reflections. How to obtain accurate AOA measurements in the presence of multipath and shadowing errors has been a subject of intensive research. AOA measurements rely on a direct line-of-sight (LOS) path from the transmitter to the receiver. However a multipath component may appear as a signal arriving from an entirely different direction and can lead to very large errors in AOA measurements. Multipath problems in AOA measurements can be addressed by using the maximum likelihood (ML) algorithms [7]. Different ML algorithms have been proposed in the literature which make different assumptions about the statistical characteristics of the incident signals [8]-[10]. They can be classified into deterministic and stochastic ML methods. Typically ML methods will estimate the AOA of each separate path in a multipath environment. The implementation of these methods is computationally intensive and requires complex multidimensional search. The dimensionality of the search is equal to the total number of paths taken by all the received signals [7]. The problem is further complicated by the fact that the total number of paths is not known a priori and must be estimated. Different from 
the earlier ML methods, which assume the incoming signal is an unknown stochastic process, another class of ML methods [11]-[13] assume that the structure of the signal waveform is known to the receiver. This assumption is possible in some digital communication systems because the modulation format is known to the receiver and many systems are equipped with a known training sequence in the preamble. This extra information is exploited to improve the accuracy of AOA measurements or simplify computation.

Yet another class of AOA measurement methods is based on so-called subspace-based algorithms [14]-[17]. The most well known methods in this category are MUSIC (multiple signal classification) [14] and ESPRIT (estimation of signal parameters by rotational invariance techniques) [15], [16]. These eigenanalysis based direction finding algorithms utilize a vector space formulation, which takes advantage of the underlying parametric data model for the sensor array problem. They require a multi-array antenna in order to form a correlation matrix using signals received by the array. The measured signal vectors received at the $M$ array elements is visualized as a vector in $M$ dimensional space. Utilizing an eigen-decomposition of the correlation matrix, the vector space is separated into signal and noise subspaces. Then the MUSIC algorithm searches for nulls in the magnitude squared of the projection of the direction vector onto the noise subspace. The nulls are a function of angle-of-arrival, from which angle-of-arrival can be estimated. For linear arrays, Root-MUSIC [18], a polynomial rooting version of MUSIC, improves the resolution capabilities of MUSIC. A weighted norm version of MUSIC, WMUSIC [19], also gives an extension in the resolution capabilities compared to the original MUSIC. ESPRIT [15], [16] is based on the estimation of signal parameters via rotational invariance techniques. It uses two displaced subarrays of matched sensor doublets to exploit an underlying rotational invariance among signal subspaces for such an array. A comprehensive experimental evaluation of MUSIC, Root-MUSIC, WMUSIC, Min-Norm [20] and ESPRIT algorithms can be found in [21]. A very large number of AOA measurement techniques have been developed which are based on MUSIC and ESPRIT, to cite but two, see e.g., [17], [22]. Due to space limitations, we do not provide an exhaustive list of them in this paper. Readers may refer to [23] for a detailed technical discussion on AOA measurement techniques.

\section{B. Distance related measurements}

Distance related measurements include propagation time based measurements, i.e., one-way propagation time measurements, roundtrip propagation time measurements and timedifference-of-arrival (TDOA) measurements, and RSS measurements. Another interesting technique measuring distance, which does not fall into the above categories, is the lighthouse approach shown in [24]. In the following paragraphs we provide further details of these techniques.

1) One-way propagation time and roundtrip propagation time measurements : One-way propagation time and roundtrip propagation time measurements are also generally known as time-of-arrival measurements. Distances between neighboring sensors can be estimated from these propagation time measurements.

One-way propagation time measurements measure the difference between the sending time of a signal at the transmitter and the receiving time of the signal at the receiver. It requires the local time at the transmitter and the local time at the receiver to be accurately synchronized. This requirement may add to the cost of sensors by demanding a highly accurate clock and/or increase the complexity of the sensor network by demanding a sophisticated synchronization mechanism. This disadvantage makes one-way propagation time measurements a less attractive option than measuring roundtrip time in WSNs. Roundtrip propagation time measurements measure the difference between the time when a signal is sent by a sensor and the time when the signal returned by a second sensor is received at the original sensor. Since the same clock is used to compute the roundtrip propagation time, there is no synchronization problem. The major error source in roundtrip propagation time measurements is the delay required for handling the signal in the second sensor. This internal delay is either known via a priori calibration, or measured and sent to the first sensor to be subtracted. A detailed discussion on circuitry design for roundtrip propagation time measurements can be found in [25].

Time delay measurement is a relatively mature field. The most widely used method for obtaining time delay measurement is the generalized cross-correlation method [26], [27]. A detailed discussion on the cross-correlation method is given in Section II-B.3.

Based on the observation that the speed of sound in the air is much smaller than the speed of light (RF) in the air, Priyantha et al. developed a technique to measure the oneway propagation time [28], which solved the synchronization problem. It uses a combination of RF and ultrasound hardware. On each transmission, a transmitter sends an RF signal and an ultrasonic pulse at the same time. The RF signal will arrive at the receiver earlier than the ultrasonic pulse. When the receiver receives the RF signal, it turns on its ultrasonic receiver and listens for the ultrasonic pulse. The time difference between the receipt of the RF signal and the receipt of the ultrasonic signal is used as an estimate of the one-way acoustic propagation time. Their method gave fairly accurate distance estimate at the cost of additional hardware and complexity of the system because ultrasonic reception suffers from severe multipath effects caused by reflections from walls and other objects.

A recent trend in propagation time measurements is the use of ultra wide band (UWB) signals for accurate distance estimation [29], [30]. A UWB signal is a signal whose bandwidth to center frequency ratio is larger than 0.2 or a signal with a total bandwidth of more than $500 \mathrm{MHz}$. UWB can achieve higher accuracy because its bandwidth is very large and therefore its pulse has a very short duration. This feature makes fine time resolution of UWB signals and easy separation of multipath signals possible.

2) Lighthouse approach to distance measurements : Another interesting approach to distance measurements is the 
lighthouse approach [24] which derives the distance between an optical receiver and a transmitter of a parallel rotating optical beam by measuring the time duration that the receiver dwells in the beam. Fig. 3 illustrates the principle of the lighthouse approach. A transmitter located at the origin is equipped with a parallel optical beam, i.e., an optical beam whose beam width $b$ is constant with respect to the distance from the rotational axis of the beam. The optical beam rotates at an unknown angular velocity $\omega$ around the $Z$ axis. An optical receiver in the XY plane and at a distance $d_{1}$ from the $Z$ axis detects the beam for a time duration $t_{1}$. From Fig. 3 , it can be shown that

$$
d_{1} \approx \frac{b}{2 \sin \left(\alpha_{1} / 2\right)}=\frac{b}{2 \sin \left(\omega t_{1} / 2\right)}
$$

The unknown angular velocity $\omega$ can be derived from the difference between the time instant when the optical receiver first detects the beam and the time instant when the optical receiver detects the beam for the second time. Therefore the distance $d_{1}$ can be derived from the time duration $t_{1}$ that the optical receiver dwells in the beam.

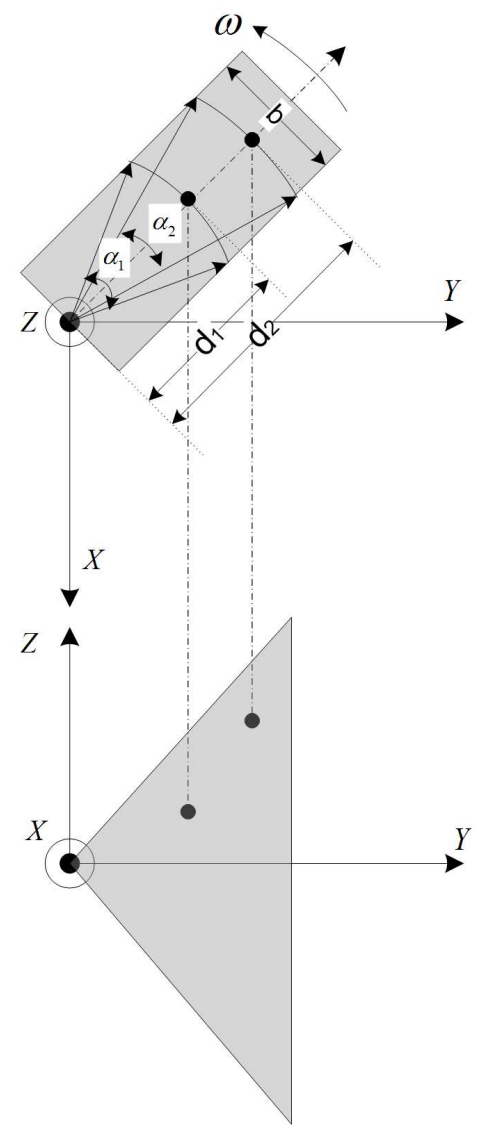

Fig. 3. An illustration of the lighthouse approach for distance measurement.

The lighthouse approach measures the distance between an optical receiver and the rotational axis of the optical beam generated by the transmitter. A major advantage of the lighthouse approach is the optical receiver can be of a very small size, thus making the idea of "smart dust" possible [24]. However the transmitter may be large. The approach also requires a direct line-of-sight between the optical receiver and the transmitter.

3) Time-difference-of-arrival measurements : There is a category of localization algorithms utilizing TDOA measurements of the transmitter's signal at a number of receivers with known location information to estimate the location of the transmitter. Fig. 4 shows a TDOA localization scenario with a group of four receivers at locations $\mathbf{r}_{1}, \mathbf{r}_{2}, \mathbf{r}_{3}, \mathbf{r}_{4}$ and a transmitter at $\mathbf{r}_{t}$. The TDOA between a pair of receivers $i$ and

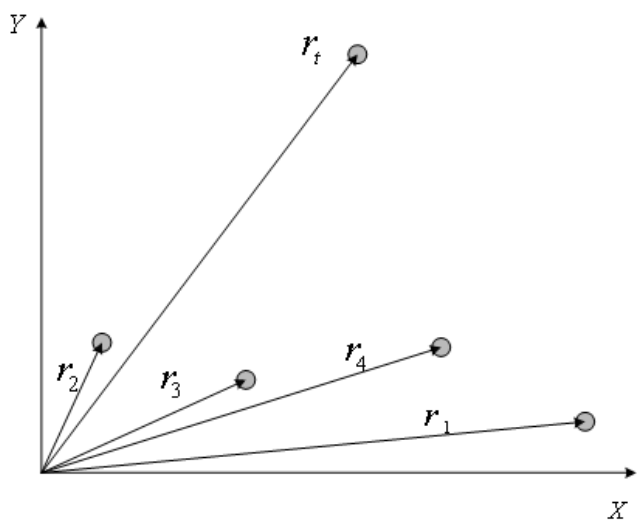

Fig. 4. Localization using time-difference-of-arrival measurements.

$j$ is given by:

$$
\triangle t_{i j} \triangleq t_{i}-t_{j}=\frac{1}{c}\left(\left\|\mathbf{r}_{i}-\mathbf{r}_{t}\right\|-\left\|\mathbf{r}_{j}-\mathbf{r}_{t}\right\|\right), \quad i \neq j
$$

where $t_{i}$ and $t_{j}$ are the time when a signal is received at receivers $i$ and $j$ respectively, $c$ is the propagation speed of the signal, and $\|\cdot\|$ denotes the Euclidean norm.

Measuring the TDOA of a signal at two receivers at separate locations is a relatively mature field [31]. The most widely used method is the generalized cross-correlation method, where the cross-correlation function between two signals $s_{i}$ and $s_{j}$ received at receivers $i$ and $j$ is given by integrating the lag product of two received signals for a sufficiently long time period $T$,

$$
\rho_{i, j}(\tau)=\frac{1}{T} \int_{0}^{T} s_{i}(t) s_{j}(t-\tau) d t
$$

The cross-correlation function can also be obtained from an inverse Fourier transform of the estimated frequency domain cross-spectral density function. Frequency domain processing is often preferred because the signals can be filtered prior to computation of the cross-correlation function. The crosscorrelation approach requires very accurate synchronization among receivers but does not impose any requirement on the signal transmitted by the transmitter. The accuracy and temporal resolution capabilities of TDOA measurements will improve when the separation between receivers increases because this increases differences between time-of-arrival. Closely spaced multiple receivers may give rise to multiple received signals that cannot be separated. For example, TDOA of multiple signals that are not separated by more than the width of their cross-correlation peaks (whose location on the time-delay axis corresponds to TDOA) usually cannot be resolved by conventional TDOA measurement techniques [32]. 
Yet another factor affecting the accuracy of TDOA measurement is multipath. Overlapping cross-correlation peaks due to multipath often cannot be resolved. Even if distinct peaks can be resolved, a method must be designed for selecting the correct peak value, such as choosing the largest or the first peak [7].

It is worth noting that Gardner et al. proposed an approach in [32] and [33], which exploits the cyclostationarity property of a certain signal to obtain substantial tolerance to noise and interference. The cyclostationarity property is a direct result of the underlying periodicities in the signal due to periodic sampling, scanning, modulating, multiplexing, and coding operations employed in the transmitter. Both the frequencyshifted and time-shifted cross-correlations are utilized to exploit the unique cyclostationarity property of the signal. Their method requires the signal of interest to have a known analog frequency or digital keying rate that is distinct from that of the interfering signal.

4) Distance estimation via received signal strength measurements : Another category of distance related measurement techniques estimates the distances between neighboring sensors from the received signal strength measurements [34][38]. These techniques are based on a standard feature found in most wireless devices, a received signal strength indicator (RSSI). They are attractive because they require no additional hardware, and are unlikely to significantly impact local power consumption, sensor size and thus cost.

In free space, other things being equal the RSS varies as the inverse square of the distance $d$ between the transmitter and the receiver. Let us denote this received power by $P_{r}(d)$. The received power $P_{r}(d)$ is related to the distance $d$ through the Friis equation [39]:

$$
P_{r}(d)=\frac{P_{t} G_{t} G_{r} \lambda^{2}}{(4 \pi)^{2} d^{2}}
$$

where $P_{t}$ is the transmitted power, $G_{t}$ is the transmitter antenna gain, $G_{r}$ is the receiver antenna gain and $\lambda$ is the wavelength of the transmitter signal in meters.

The free-space model however is an over-idealization, and the propagation of a signal is affected by reflection, diffraction and scattering. Of course, these effects are environment (indoors, outdoors, rain, buildings, etc.) dependent. However, it is accepted on the basis of empirical evidence that it is reasonable to model the $\operatorname{RSS} P_{r}(d)$ at any value of $d$ at a particular location as a random and log-normally distributed random variable with a distance-dependent mean value [40], [41]. That is,

$$
P_{r}(d)[d B m]=P_{0}\left(d_{0}\right)[d B m]-10 n_{p} \log _{10}\left(\frac{d}{d_{0}}\right)+X_{\sigma}
$$

where $P_{0}\left(d_{0}\right)[d B m]$ is a known reference power value in $\mathrm{dB}$ milliwatts at a reference distance $d_{0}$ from the transmitter, $n_{p}$ is the path loss exponent that measures the rate at which the RSS decreases with distance and the value of $n_{p}$ depends on the specific propagation environment, $X_{\sigma}$ is a zero mean Gaussian distributed random variable with standard deviation $\sigma$ and it accounts for the random effect of shadowing [39]. In this paper, we use the notation $[\mathrm{dBm}]$ to denote that power is in $\mathrm{dB}$ milliwatts units. Otherwise, it is in milliwatts.

It is trivial to conclude from Eq. 6 that, given the RSS measurement, $P_{i j}$, between a transmitter $i$ and a receiver $j$, a maximum likelihood estimate of the distance, $d_{i j}$, between the transmitter and the receiver is:

$$
\hat{d}_{i j}=d_{0}\left(\frac{P_{i j}}{P_{0}\left(d_{0}\right)}\right)^{-1 / n_{p}}
$$

Note that $P_{i j}$ and $P_{0}\left(d_{0}\right)$ in Eq. 7 are measured in milliwatts instead of $\mathrm{dB}$ milliwatts. Using Eq. 6 and Eq. 7, the estimated distance $\hat{d}_{i j}$ can be related to the true distance:

$$
\hat{d}_{i j}=d_{i j} 10^{-\frac{X_{\sigma}}{10 n_{p}}}=d_{i j} 10^{-\frac{\ln (10) X_{\sigma}}{10 n_{p} \ln (10)}}=d_{i j} e^{-\frac{X_{\sigma}}{\eta n_{p}}}
$$

where $\eta=\frac{10}{\ln (10)}$. The expected value of $\hat{d}_{i j}$ is:

$$
E\left(\hat{d}_{i j}\right)=\frac{1}{\sqrt{2 \pi} \sigma} \int_{-\infty}^{\infty} d_{i j} e^{-\frac{X_{\sigma}}{\eta n_{p}}} e^{-\frac{X_{\sigma}}{2 \sigma^{2}}} d X_{\sigma}=d_{i j} e^{\frac{\sigma^{2}}{2 \eta^{2} n_{p}^{2}}}
$$

Thus the maximum likelihood estimate in Eq. 7 is a biased estimate of the true distance and an unbiased estimate is given by:

$$
\hat{d}_{i j}=d_{0}\left(\frac{P_{i j}}{P_{0}\left(d_{0}\right)}\right)^{-1 / n_{p}} e^{-\frac{\sigma^{2}}{2 \eta^{2} n_{p}^{2}}}
$$

\section{RSS profiling measurements}

Yet another category of localization techniques, i.e., the RSS profiling-based localization techniques [42]-[46], work by constructing a form of map of the signal strength behavior in the coverage area. The map is obtained either offline by a priori measurements or online using sniffing devices [44] deployed at known locations. They have been mainly used for location estimation in WLANs, but they would appear to be attractive also for wireless sensor networks.

In this technique, in addition to there being anchor nodes (e.g., access points in WLANs) and non-anchor nodes, a large number of sample points (e.g., sniffing devices) are distributed throughout the coverage area of the sensor network. At each sample point, a vector of signal strengths is obtained, with the $j^{\text {th }}$ entry corresponding to the $j^{\text {th }}$ anchor's transmitted signal. Of course, many entries of the signal strength vector may be zero or very small, corresponding to anchor nodes at larger distances (relative to the transmission range or sensing radius) from the sample point. The collection of all these vectors provides (by extrapolation in the vicinity of the sample points) a map of the whole region. The collection constitutes the RSS model, and it is unique with respect to the anchor locations and the environment. The model is stored in a central location. By referring to the RSS model, a non-anchor node can estimate its location using the RSS measurements from anchors.

In summary, a number of measurement techniques are available for WSN localization. Which measurement technique to use for location estimation will depend on the specific application. Typically, localization algorithms based on AOA and propagation time measurements are able to achieve better accuracy than localization algorithms based on RSS measurements. However, that accuracy is achieved at the expense 
of higher equipment cost. Patwati et al. gave the CramérRao lower bounds for location estimation using TOA, RSS and AOA measurements respectively in [5]. However the Cramér-Rao lower bound may be too optimistic when the measurement error deviates from Gaussian. Moreover the Cramér-Rao bound assumes the underlying estimator is an unbiased estimator. This assumption may not be satisfied by many localization techniques.

\section{ONE-HOP LOCALIZATION TECHNIQUES}

In this section, we discuss the principles of one-hop localization techniques in which the non-anchor node to be localized is the one-hop neighbor of a sufficient number of anchors.

\section{A. AOA based one-hop localization techniques}

In the absence of noise and interference, bearing lines from two or more receivers will intersect to determine a unique location, i.e., the location estimate of the transmitter. In the presence of noise, more than two bearing lines will not intersect at a single point and statistical algorithms, sometimes called triangulation or fixing methods, are required in order to obtain the location estimate of the transmitter [47], [48]. This is shown in Fig. 5

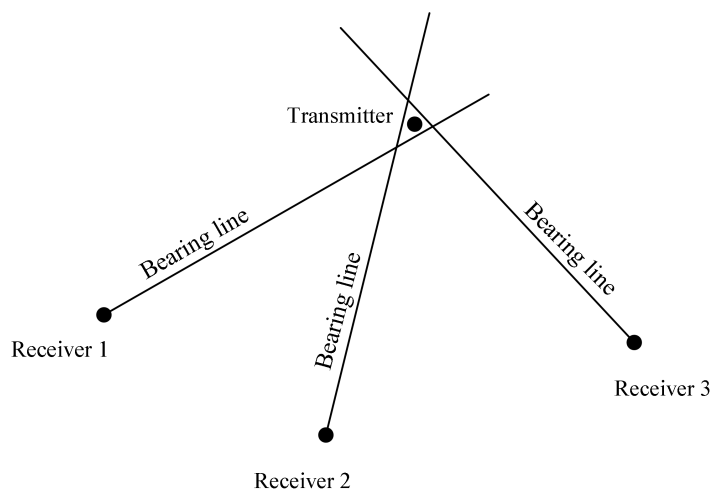

Fig. 5. In the presence of noise, bearing lines from three receivers will not interact at the same point.

Location estimation using bearing measurements is a well researched problem [47]-[52]. The pioneering work in the area is that of Stansfield [49]. His approach has been further generalized in [50], [52] and has been implemented in many practical systems. Another well-known approach is the maximum likelihood estimator [47], [51].

The 2D localization problem using bearing measurements can be formulated as follows. Let $\mathbf{x}_{t}=\left[x_{t}, y_{t}\right]^{T}$ be the true coordinate vector of the transmitter to be estimated from bearing measurements $\beta=\left[\beta_{1}, \ldots, \beta_{N}\right]^{T}$, where $N$ is the total number of receivers. Let $\mathbf{x}_{i}=\left[x_{i}, y_{i}\right]^{T}$ be the known location of the receiver associated with the $i^{\text {th }}$ bearing measurement. Denote by $\theta(\mathbf{x})=\left[\theta_{1}(\mathbf{x}), \ldots, \theta_{N}(\mathbf{x})\right]^{T}$ the bearings of a transmitter located at $\mathbf{x}=[x, y]^{T}$ at the receiver locations, where $\theta_{i}(\mathbf{x}), 1 \leq i \leq N$ is related to $\mathbf{x}$ by:

$$
\tan \theta_{i}(\mathbf{x})=\frac{y-y_{i}}{x-x_{i}}
$$

The measured bearings of the transmitter consist of the true bearings corrupted by additive noises $\varepsilon=\left[\varepsilon_{1}, \ldots, \varepsilon_{N}\right]^{T}$, which are assumed to be zero-mean Gaussian noises with $N \times N$ covariance matrices $\mathbf{S}=\operatorname{diag}\left\{\sigma_{1}^{2}, \ldots, \sigma_{N}^{2}\right\}$, i.e.,

$$
\beta=\theta\left(\mathbf{x}_{t}\right)+\varepsilon
$$

When the receivers are identical and much closer to each other than to the transmitter, the variances of bearing measurement errors are equal, i.e., $\sigma_{1}^{2}=\cdots \sigma_{N}^{2}=\sigma^{2}$. The ML estimator of the transmitter location $\mathbf{x}_{t}$ is given by:

$$
\begin{aligned}
\hat{\mathbf{x}}_{t} & =\arg \min \frac{1}{2}\left[\theta\left(\hat{\mathbf{x}}_{t}\right)-\beta\right]^{T} \mathbf{S}^{-1}\left[\theta\left(\hat{\mathbf{x}}_{t}\right)-\beta\right] \\
& =\arg \min \frac{1}{2} \sum_{i=1}^{N} \frac{\left(\theta_{i}\left(\hat{\mathbf{x}}_{t}\right)-\beta_{i}\right)^{2}}{\sigma_{i}^{2}}
\end{aligned}
$$

The nonlinear minimization problem in Eq. 13 can be solved by a Newton-Gauss iteration [47], [48]:

$$
\begin{aligned}
& \hat{\mathbf{x}}_{t, k+1}=\hat{\mathbf{x}}_{t, k}+ \\
& \left(\theta_{\mathbf{x}}\left(\hat{\mathbf{x}}_{t, k}\right)^{T} \mathbf{S}^{-1} \theta_{\mathbf{x}}\left(\hat{\mathbf{x}}_{t, k}\right)\right)^{-1} \theta_{\mathbf{x}}\left(\hat{\mathbf{x}}_{t, k}\right)^{T} \mathbf{S}^{-1}\left[\beta-\theta_{\mathbf{x}}\left(\hat{\mathbf{x}}_{t, k}\right)\right]
\end{aligned}
$$

where $\theta_{\mathbf{x}}\left(\hat{\mathbf{x}}_{t, k}\right)$ denotes the partial derivative of $\theta$ with respect to $\mathbf{x}$ evaluated at $\hat{\mathbf{x}}_{t, k}$. The use of Eq. 15 requires an initial estimate close enough to the true minimum of the cost function. Such an initial estimate may be obtained from prior information, or using a suboptimal procedure [48].

The Stansfield approach assumes that the measurement error is small enough such that $\varepsilon_{i} \approx \sin \varepsilon_{i}, 1 \leq i \leq N$. In that case, the cost function in Eq. 14 becomes:

$$
\frac{1}{2} \sum_{i=1}^{N} \frac{\sin ^{2}\left(\theta_{i}\left(\hat{\mathbf{x}}_{t}\right)-\beta_{i}\right)}{\sigma_{i}^{2}}
$$

Using the relation

$$
\begin{aligned}
\sin \left(\theta_{i}\left(\mathbf{x}_{t}\right)-\beta_{i}\right) & =\sin \theta_{i}\left(\mathbf{x}_{t}\right) \cos \beta_{i}-\cos \theta_{i}\left(\mathbf{x}_{t}\right) \sin \beta_{i} \\
& =\frac{\left(y_{t}-y_{i}\right) \cos \beta_{i}-\left(x_{t}-x_{i}\right) \sin \beta_{i}}{r_{i}}
\end{aligned}
$$

where $r_{i}=\sqrt{\left(x_{t}-x_{i}\right)^{2}+\left(y_{t}-y_{i}\right)^{2}}$, Eq. 16 becomes

$$
\begin{aligned}
& \frac{1}{2} \sum_{i=1}^{N} \frac{\left[\left(y_{t}-y_{i}\right) \cos \beta_{i}-\left(x_{t}-x_{i}\right) \sin \beta_{i}\right]^{2}}{\sigma_{i}^{2} r_{i}^{2}} \\
= & \frac{1}{2}\left(\mathbf{A} \mathbf{x}_{t}-\mathbf{b}\right)^{T} \mathbf{R}^{-1} \mathbf{S}^{-1}\left(\mathbf{A} \mathbf{x}_{t}-\mathbf{b}\right)
\end{aligned}
$$

where

$$
\begin{aligned}
& \mathbf{A}= {\left[\begin{array}{cc}
\sin \beta_{1} & -\cos \beta_{1} \\
\vdots & \vdots \\
\sin \beta_{N} & -\cos \beta_{N}
\end{array}\right] } \\
& \mathbf{b}=\left[\begin{array}{c}
x_{1} \sin \beta_{1}-y_{1} \cos \beta_{1} \\
\vdots \\
x_{N} \sin \beta_{1}-y_{1} \cos \beta_{N}
\end{array}\right] \\
& \mathbf{R}=\operatorname{diag}\left\{r_{1}^{2}, \cdots, r_{N}^{2}\right\}
\end{aligned}
$$

Stansfield implicitly assumes that even though $\mathbf{R}$ is not perfectly known, a rough estimate of $\mathbf{R}$ can be obtained. Since the cost function weakly depends on $\mathbf{R}$, the fact that the estimate 
is rough will not significantly affect the solution. Under these assumptions, the minimization of Eq. 17 with respect to $\mathbf{x}_{t}$ is a well known problem and the solution is given by:

$$
\hat{\mathbf{x}}_{t}=\left(\mathbf{A}^{T} \mathbf{R}^{-1} \mathbf{S}^{-1} \mathbf{A}\right)^{-1} \mathbf{A}^{T} \mathbf{R}^{-1} \mathbf{S}^{-1} \mathbf{b}
$$

Note that the closed form solution in the Stansfield approach depends on two assumptions: first, the measurement error is small such that $\varepsilon_{i} \approx \sin \varepsilon_{i}, 1 \leq i \leq N$; second, $\mathbf{R}$ is known. One may chose to accept the first assumption but reject the second assumption. In that case an iterative procedure can be used to obtain the solution to the minimization problem, which has no advantage over the ML technique [48].

Analytical expressions for the bias and the covariance matrix of the estimation errors associated with the ML approach and with the Stansfield approach were given in [48]. It was shown that the Stansfield approach provides biased estimates even for a large number of bearing measurements and the ML approach is asymptotically unbiased at a large number of measurements. However the RMS (root mean square) error of Stansfield approach is not necessarily larger than that of the ML approach. A quite different approach is referred to at the end of Section III-C, using a very recently introduced method of exploiting the over-determined nature of the noiseless problem.

\section{B. TDOA-based one-hop localization techniques}

Given the TDOA measurement $\triangle t_{i j}$ and the coordinates of receivers $i$ and $j$, Eq. 3 defines one branch of a hyperbola whose foci are at the locations of receivers $i$ and $j$ and on which the transmitter $\mathbf{r}_{t}$ must lie. In $\Re^{2}$, measurements from a minimum of three receivers are required to uniquely determine the location of the transmitter. This is illustrated in Fig. 6.

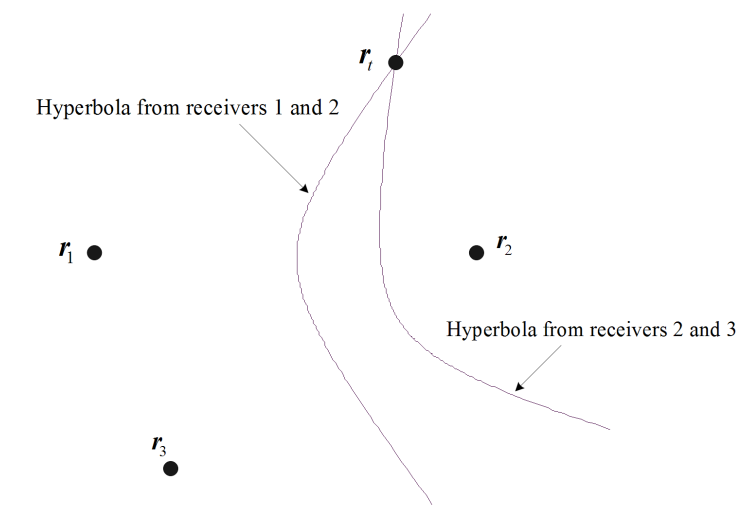

Fig. 6. Intersecting hyperbolas from three receivers.

In a system of $N$ receivers, there are $N-1$ linearly independent TDOA equations, which can be written compactly as:

$$
\left[\begin{array}{c}
\left\|\mathbf{r}_{1}-\mathbf{r}_{t}\right\|-\left\|\mathbf{r}_{N}-\mathbf{r}_{t}\right\|-c \Delta t_{1 N} \\
\vdots \\
\left\|\mathbf{r}_{N-1}-\mathbf{r}_{t}\right\|-\left\|\mathbf{r}_{N}-\mathbf{r}_{t}\right\|-c \Delta t_{N-1 N}
\end{array}\right]=\mathbf{0}
$$

In practice, $\triangle t_{i j}$ is not available; instead we have the noisy TDOA measurement $\triangle \tilde{t}_{i j}$ given by:

$$
\triangle \tilde{t}_{i j}=\triangle t_{i j}+n_{i j}
$$

where $n_{i j}$ denotes an additive noise, which is usually assumed to be an independent zero-mean Gaussian distributed random variable. Eq. 22 is a nonlinear equation that is difficult to solve, especially when the receivers are arranged in an arbitrary fashion. Moreover, in the presence of noise, Eq. 22 may not have a solution.

A noisy version of Eq. 22 can be written as:

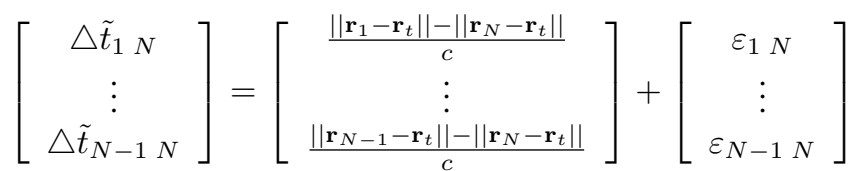

Denote by $\triangle \tilde{\mathbf{t}}$ the TDOA measurement vector $\left[\triangle \tilde{t}_{1 N}, \ldots, \triangle \tilde{t}_{N-1 N}\right]^{T}$. Denote by $\mathbf{f}\left(\mathbf{r}_{\mathbf{t}}\right)$ the vector $\left[\frac{1}{c}\left(\left\|\mathbf{r}_{1}-\mathbf{r}_{t}\right\|-\left\|\mathbf{r}_{N}-\mathbf{r}_{t}\right\|\right), \quad \ldots\right.$, $\left.\frac{1}{c}\left(\left\|\mathbf{r}_{N-1}-\mathbf{r}_{t}\right\|-\left\|\mathbf{r}_{N}-\mathbf{r}_{t}\right\|\right)\right]^{T}$ and denote by $\mathbf{S}$ the covariance matrix of the TDOA measurement errors. The ML estimator minimizes the following quadratic function:

$$
Q\left(\hat{\mathbf{r}}_{t}\right)=\left[\triangle \tilde{\mathbf{t}}-\mathbf{f}\left(\hat{\mathbf{r}}_{\mathbf{t}}\right)\right]^{T} \mathbf{S}^{-1}\left[\triangle \tilde{\mathbf{t}}-\mathbf{f}\left(\hat{\mathbf{r}}_{\mathbf{t}}\right)\right]
$$

in which $\mathbf{f}\left(\mathbf{r}_{\mathbf{t}}\right)$ is a nonlinear vector function. In order to obtain a reasonably simple estimator, $\mathbf{f}\left(\mathbf{r}_{\mathbf{t}}\right)$ can be linearized using Taylor series around a reference point $\mathbf{r}_{\mathbf{0}}$ :

$$
\mathbf{f}\left(\mathbf{r}_{\mathbf{t}}\right) \approx \mathbf{f}\left(\mathbf{r}_{\mathbf{0}}\right)+\mathbf{f}_{\mathbf{r}}\left(\mathbf{r}_{\mathbf{0}}\right)\left(\mathbf{r}_{\mathbf{t}}-\mathbf{r}_{\mathbf{0}}\right)
$$

where $\mathbf{f}_{\mathbf{r}}\left(\mathbf{r}_{\mathbf{0}}\right)$ is a $(N-1) \times 2\left(\right.$ in $\left.\Re^{2}\right)$ matrix of partial derivative of $\mathbf{f}$ with respect to $\mathbf{r}$ evaluated at $\mathbf{r}_{0}$. A recursive solution to the ML estimator can then be obtained [47]:

$$
\begin{aligned}
& \hat{\mathbf{r}}_{t, k+1}=\hat{\mathbf{r}}_{t, k}+ \\
& \left(\mathbf{f}_{\mathbf{r}}\left(\mathbf{r}_{\mathbf{t}, \mathbf{k}}\right)^{T} S^{-1} \mathbf{f}_{\mathbf{r}}\left(\mathbf{r}_{\mathbf{t}, \mathbf{k}}\right)\right)^{-1} \mathbf{f}_{\mathbf{r}}\left(\mathbf{r}_{\mathbf{t}, \mathbf{k}}\right)^{T} S^{-1}\left[\triangle \tilde{\mathbf{t}}-\mathbf{f}\left(\mathbf{r}_{\mathbf{t}, \mathbf{k}}\right)\right]
\end{aligned}
$$

This method relies on a good initial guess of the transmitter location. Moreover, in some situations this method can result in significant location estimation errors due to geometric dilution of precision (GDOP) effects. GDOP describes a situation in which a relatively small ranging error can result in a large location estimation error because the transmitter is located on a portion of the hyperbola far away from both receivers [7], [53]. Fang [54] gave an exact solution to the hyperbolic equations in Eq. 22 when the number of TDOA measurements are equal to the number of unknown transmitter coordinates. However his solution cannot make use of extra measurements. Other techniques that can deal with the more general situation with extra measurements include the spherical interpolation method [55], which is derived from least-squares "equationerror" minimization, and the divide and conquer method [56]. The divide and conquer estimate is formed by combining the maximum likelihood estimates using possibly overlapping subsections of the measurement data vector. The divide and conquer method can achieve the optimum performance but it requires that the Fisher information matrix is sufficiently large. Chan et al. [57] developed a closed form solution valid for an arbitrary number of TDOA measurements and arbitrarily distributed transmitters. The solution is an approximation of the maximum likelihood estimator when the TDOA measurement errors are small. Chan's method performs significantly 
better than the spherical interpolation method and is more robust against noise than the divide and conquer method. The computational complexity of Chan's method is comparable to the spherical interpolation method but substantially less than the Taylor-series method [47]. Recently, Doğançay and Drake et al. developed a closed form solution for localization of distant transmitters based on triangulation of hyperbolic asymptotes [58], [59]. The hyperbolic curves are approximated by linear asymptotes. The solution exhibits some performance degradation with respect to the maximum likelihood estimator at low noise levels but outperforms the maximum likelihood estimator at medium to high noise levels.

\section{Distance-based one-hop localization techniques}

The most well-known distance-based localization technique is based on use of GPS. The GPS space segment consists of 24 satellites in the medium earth orbit at a nominal altitude of $20,200 \mathrm{~km}$ with an orbital inclination of $55^{0}$. Each satellite carries several high accuracy atomic clocks and radiates a sequence of bits that starts at a precisely known time. The location of a GPS satellite at any particular time instant is known. A GPS receiver located on the earth derives its distance to a GPS satellite from the difference of the time a GPS signal is received at the receiver and the time the GPS signal is radiated by the GPS satellite. Ideally, distance measurements to three GPS satellites allow the GPS receiver to uniquely determine its position. In reality, four satellites, rather than three, are required because of synchronization error in the receiver's clock. The fourth distance measurement provides information from which the synchronization error of the receiver can be corrected and the receiver's clock can be synchronized to an accuracy better than 100ns.

Generally in a WSN, for a non-anchor node at unknown location $\mathbf{x}_{t}$ with noise-contaminated distance measurements $\tilde{d}_{1}, \ldots, \tilde{d}_{N}$ to $N$ anchors at known locations $\mathbf{x}_{1}, \ldots, \mathbf{x}_{N}$, the location estimation problem can be formulated using a maximum likelihood approach as:

$$
\hat{\mathbf{x}}_{t}=\arg \min \left[\mathbf{d}\left(\hat{\mathbf{x}}_{t}\right)-\tilde{\mathbf{d}}\right]^{T} \mathbf{S}^{-1}\left[\mathbf{d}\left(\hat{\mathbf{x}}_{t}\right)-\tilde{\mathbf{d}}\right]
$$

where $\tilde{\mathbf{d}}$ is a $N \times 1$ distance measurement vector, $\mathbf{d}\left(\hat{\mathbf{x}}_{t}\right)$ is also a $N \times 1$ vector $\left[\left\|\hat{\mathbf{x}}_{t}-\mathbf{x}_{1}\right\|, \ldots,\left\|\hat{\mathbf{x}}_{t}-\mathbf{x}_{N}\right\|\right]$ and $\mathbf{S}$ is the covariance matrix of the distance measurement errors. This minimization problem can be solved using a similar procedure described in Section III-A and Section III-B.

An interesting development in the area is the use of the Cayley-Menger determinant [60], [61] to reduce the impact of distance measurement errors on the location estimate [62], [63]. To illustrate the concept, consider a non-anchor node $\mathbf{x}_{t}$ having distance measurements to three anchors $\mathbf{x}_{1}, \mathbf{x}_{2}, \mathbf{x}_{3}$ in $\Re^{2}$, which is shown in Fig. 7.

The Cayley-Menger determinant of this quadrilateral is given by:

$$
D\left(\mathbf{x}_{1}, \mathbf{x}_{2}, \mathbf{x}_{3}, \mathbf{x}_{t}\right)=\left|\begin{array}{ccccc}
0 & d_{12}^{2} & d_{13}^{2} & d_{t 1}^{2} & 1 \\
d_{12}^{2} & 0 & d_{23}^{2} & d_{t 2}^{2} & 1 \\
d_{13}^{2} & d_{23}^{2} & 0 & d_{t 3}^{2} & 1 \\
d_{t 1}^{2} & d_{t 2}^{2} & d_{t 3}^{2} & 0 & 1 \\
1 & 1 & 1 & 1 & 0
\end{array}\right|
$$

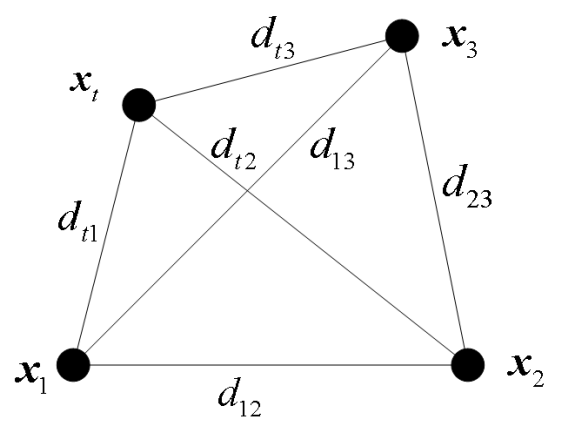

Fig. 7. A fully-connected planar quadrilateral in sensor networks.

A classical result on the Cayley-Menger determinant is given by the following theorem:

Theorem 1: (Theorem 112.1 in [61]) Consider an n-tuple of points $\mathbf{x}_{1}, \ldots, \mathbf{x}_{n}$ in $m$-dimensional space with $n \geq m+1$. The rank of the Cayley-Menger matrix $M\left(\mathbf{x}_{1}, \ldots, \mathbf{x}_{n}\right)$ (defined analogously to the right side of Eq. 29 but without the determinant operation) is at most $m+1$.

According to the above theorem, in $\Re^{2}$,

$$
D\left(\mathbf{x}_{1}, \mathbf{x}_{2}, \mathbf{x}_{3}, \mathbf{x}_{t}\right)=0
$$

Note that the distances between anchors $d_{12}, d_{13}$ and $d_{23}$ can be inferred from known anchor positions. The true distances between the non-anchor node and the anchors are related to the measured distances by:

$$
\tilde{d}_{t i}=d_{t i}+\varepsilon_{i}, 1 \leq i \leq 3
$$

Putting Eq. 31 into Eq. 30, it can be shown that [62]:

$$
\varepsilon^{T} A \varepsilon+\varepsilon^{T} b+c=0
$$

where $\varepsilon=\left[\varepsilon_{1}, \varepsilon_{2}, \varepsilon_{3}\right]^{T}$, the matrix $A$, vectors $b$ and $c$ can be expressed in the form of known inter-anchor distances $d_{12}, d_{13}, d_{23}$ and measured distances $\tilde{d}_{t 1}, \tilde{d}_{t 2}, \tilde{d}_{t 3}$. Eq. 32 forms an additional equality constraint on the non-anchor node position. For a non-anchor node forming $m$ quadrilaterals with neighboring anchors, there are $m$ independent equations like Eq. 32. These equality constraints can be combined with Eq. 28 using Lagrange multipliers [62]. Numerical methods, such as the gradient descent algorithm, can be exploited to search for the solution, which gives a location estimate superior to that obtained using Eq. 28 only.

The essence of using the Cayley-Menger determinant to reduce the impact of distance measurement errors is: the six edges of a planar quadrilateral are not independent, instead they must satisfy the equality constraint in Eq. 30. This equality constraint can be exploited to reduce the impact of distance measurement errors. This idea may also extend to TDOA and AOA [64] based localization.

\section{Lighthouse approach to one-hop localization}

The lighthouse approach uses a base station equipped with three mutually perpendicular parallel optical beams to locate all optical receivers within the range and line-of-sight of the beams in $\Re^{3}$. In Section II-B.2, we have described the principle of the lighthouse approach to measure the distance of an 
optical receiver to the rotational axis of a parallel optical beam [24]. Without loss of generality, assuming the rotational axes of the three mutually perpendicular parallel optical beams are $X, Y$, and $Z$ axes respectively. As shown in Fig. 8, ignoring the measurement errors, the unknown receiver location $\mathrm{x}_{t}=$ $\left[x_{t}, y_{t}, z_{t}\right]^{T}$ is related to the distance measurements to the $X$, $Y$, and $Z$ axes, denoted by $\tilde{d}_{x}, \tilde{d}_{y}$ and $\tilde{d}_{z}$ respectively, through the following equations:

$$
\begin{aligned}
\tilde{d}_{x}^{2} & =y_{t}^{2}+z_{t}^{2} \\
\tilde{d}_{y}^{2} & =x_{t}^{2}+z_{t}^{2} \\
\tilde{d}_{z}^{2} & =x_{t}^{2}+y_{t}^{2}
\end{aligned}
$$

Solving the above equations gives eight solutions, each corresponding a point in one of the eight quadrants in $\Re^{3}$. By using priori knowledge of which quadrant the receiver is located in, only one solution is chosen.

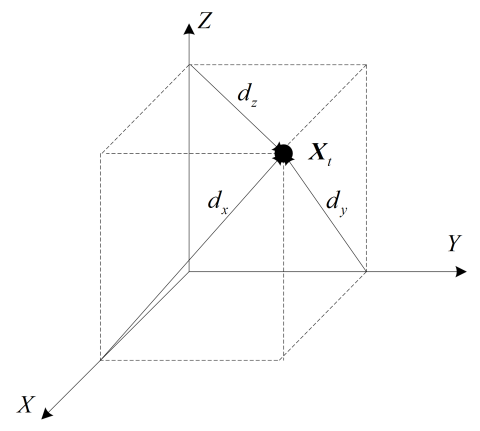

Fig. 8. An illustration of the lighthouse approach to localization.

A practical system using the lighthouse approach for $2 \mathrm{D}$ (XY plane) localization was reported to have a mean relative error of $1.1 \%$ in $x$ axis (i.e., $\frac{1}{M} \sum_{i=1}^{M}\left|\hat{x}_{t, i}-x_{t, i}\right| / x_{t, i}, M$ is the total number of receivers in the experiment) and a mean relative error of $2.8 \%$ in $y$ axis (i.e., $\frac{1}{M} \sum_{i=1}^{M}\left|\hat{y}_{t, i}-y_{t, i}\right| / y_{t, i}$ ) [24]. Techniques dealing with non-ideal situations such as misalignment of the rotational axes of optical beams and nonparallel beams were also discussed in [24].

\section{E. RSS-profiling based localization}

Given the RSS model constructed using the procedure described in Section II-C, each non-anchor node unaware of its location uses the signal strength measurements it collects, stemming from the anchor nodes within its sensing region, and thus creates its own RSS finger print, which is then transmitted to the central station. Then the central station matches the presented signal strength vector to the RSS model, using probabilistic techniques or some kind of nearest neighborbased method, which chooses the location of a sample point whose RSS vector is the closest match to that of the nonanchor node to be the estimated location of the non-anchor node [42]. In this way, an estimate of the location of the nonanchor node can be obtained. The estimate is transmitted to the non-anchor node from the central station. Obviously, a non-anchor node could also obtain the full RSS model from the central station and perform its own location estimation.

The accuracy of this technique depends on two distinct factors: the particular technique used to build the RSS model, with the resultant quality of that model, and the technique used to fit the measured signal strength vector from a non-anchor node into the appropriate part of the model. In comparison with distance-estimation based techniques, the RSS-profiling based techniques produce relatively small location estimation errors [42]. In [35], Elnahrway et al. proposed several areabased localization algorithms using RSS profiling; these algorithms are area based because instead of estimating the exact location of the non-anchor node, they simply estimate a possible area that should contain it. Two different performance parameters apply: accuracy, or the likelihood that an object is within the area, and precision, i.e., the size of the area. Reference [35] also considered three different techniques for the area based algorithms, viz., single point matching, area based probability and Bayesian networks. The performance of all three algorithms was compared with the point based algorithm of [42]. The conclusion was that all algorithms performed similarly, with a fundamental limit existing in the case of the RSS-profiling based localization algorithms, a conclusion also consistent with that of [65]. A rule of thumb is provided in [35]. Using 802.11 technology, with dense sampling and a good algorithm, one can expect a median localization error (i.e., distance between the estimated location and the true location) of about $3 \mathrm{~m}$ and a $97^{t h}$ percentile error of about $9 \mathrm{~m}$. With relatively sparse sampling, every $6 \mathrm{~m}$, or $37 \mathrm{~m}^{2} /$ sample, one can still achieve a median error of $4.5 \mathrm{~m}$ and $95^{\text {th }}$ percentile error of $12 \mathrm{~m}$.

In [66], Ni et al. presented a weighted version of the RSSprofiling based localization technique which achieves a more accurate location estimate. Denote by $\gamma$ the signal strength vector of the non-anchor node. Denote by $\beta_{i}$ and $\mathbf{x}_{i}$ the signal strength vector and the location vector of the $i^{\text {th }}$ sample point respectively. In the weighted version of the RSS-profiling based localization algorithm, the location estimate of the nonanchor point is given by:

$$
\hat{\mathbf{x}}_{t}=\sum_{i=1}^{N} \frac{\frac{1}{\left\|\gamma-\beta_{i}\right\|^{2}}}{\sum_{i=1}^{N} \frac{1}{\left\|\gamma-\beta_{i}\right\|^{2}}} \mathbf{x}_{i}
$$

where $\left\|\gamma-\beta_{i}\right\|$ denotes the Euclidean distance between the two vectors $\gamma$ and $\beta_{i}$, and $N$ is the total number of sample points. Experimental evaluation showed that Ni's approach achieves a median localization error of $1 \mathrm{~m}$ and a maximum localization error of $2 m$, which appears to be better than those reported in [67].

The major practical obstacle in the RSS-profiling based localization is the extensive amount of profiling data required. Substantial and possibly costly initial experiments are needed to establish the model. Subsequent changes in the environment (e.g. inside a building, office occupancy can change) can affect the model, and so a static model derived from a single-shot experiment may be inadequate in some applications. Recently, there has been proposed a method of online profiling, which would reduce or possibly eliminate the amount of profiling required before deployment, but at the expense of deploying a large number of additional devices (termed "sniffers") at known locations [36], [44]. Together with a large number of stationary emitters (anchor nodes) deployed at known 
locations, the "sniffers" can be used to construct and update the RSS model online.

\section{F. Localization based on hybrid measurements}

There are a number of other localization algorithms based on data fusion [68] of hybrid measurements. McGuire et al. explored data fusion of RSS and TOA measurements for mobile terminal localization in a CDMA cellular network [69]. Li et al. considered mobile user localization using hybrid TDOA/AOA measurements in a macrocell wideband CDMA system with frequency division duplex [70]. Gu et al. considered mobile user localization in a CDMA cellular network using hybrid AOA/TOA measurements [71]. Kleine-Ostmann et al. [72] presented a data fusion architecture for combining TDOA and TOA measurements. Thomas et al. considered the fusion of TDOA and AOA measurements [73]. Catovic [74] computed the Cramér-Rao bounds on the location estimation accuracy of two different hybrid schemes, i.e., TOA/RSS and TDOA/RSS, and found that hybrid schemes offer improved accuracy with respect to conventional TOA and TDOA schemes. Fundamentally, localization based on hybrid measurements can achieve a performance improvement over that based on a single measurement type because measurement noise for different types of measurements comes from different sources. Therefore errors in the location estimate for each measurement type are at least partially independent. This independence between different types of measurements can be exploited by data fusion techniques [68] to create estimators that have better accuracy than estimators based on single measurement types. Among those hybrid techniques, the fusion of RSS and TOA measurements appears to be the most attractive for a WSN because of its relatively simple hardware requirement.

\section{Nonline-OF-Sight ERRoR Mitigation}

A common problem in many localization techniques is the nonline-of-sight (NLOS) error mitigation. NLOS errors between two sensors can arise when either the line-of-sight between them is obstructed, perhaps by a building, or the lineof-sight measurements are contaminated by reflected and/or diffracted signals. As NLOS error mitigation in AOA based localization [75]-[77] and distance based localization [78]-[81] share some degree of commonality, we review them together in this section. Most NLOS error mitigation techniques assume that NLOS corrupted measurements only constitute a small fraction of the total measurements. Since NLOS corrupted measurements are inconsistent with LOS expectations, they can be treated as outliers. A typical approach is to assume that the measurement error has a Gaussian distribution, then the least-squares residuals are examined to determine if NLOS errors are present [76], [80], [81] (by regarding any large residual as due to the NLOS signals). Unfortunately, this approach fails to work when multiple NLOS measurements are present as the multiple outliers in the measurement tend to bias the final estimate decision and reduce the residuals. This behavior motivates the use of deletion diagnostics. In deletion diagnostics, the effects of eliminating various measurements from the total set are computed and ranked [80], [82].
Some other approaches are proposed to reduce estimation errors for time-of-arrival (TOA) [79], [83] and TDOA [75] respectively when the majority of the measurements are NLOS measurements. In [79], Venkatraman et al. employed a constrained nonlinear optimization approach for TOA NLOS error mitigation in a cellular network. Bounds on the NLOS error and the relationship between the true ranges are extracted from the geometry of the cell layout and the measured range circles to serve as constraints. Wang et al. proposed an algorithm which attempts to mitigate NLOS error effect in a TOA based location system, utilizing the information that NLOS error causes the measured distance to be greater than the true distance. A quadratic programming approach is used to solve for an ML estimate of the source location [84]. Cong et al. proposed two NLOS error mitigation algorithms assuming a full knowledge of NLOS error distribution (i.e., the probability that each measurement is NLOS and the probability distribution of NLOS error) and a partial knowledge of NLOS error distribution (i.e., the probability that each measurement is NLOS and the mean value of the probability distribution of NLOS error) respectively [75]. However this prior information may be difficult to obtain in a WSN.

\section{Connectivity Based Multihop Localization ALGORITHMS}

In the following sections, we shall review multihop localization techniques in which the non-anchor nodes are not necessarily the one-hop neighbors of the anchors. In particular, we focus on connectivity-based and distance-based multihop localization algorithms due to their prevalence in multihop WSN localization techniques.

There is a distinct category of localization algorithms, called connectivity-based or "range free" localization algorithms, which do not rely on any of the measurement techniques in the earlier sections. Instead they use the connectivity information, i.e., "who is within the communications range of whom" [85] to estimate the locations of the non-anchor nodes. The principle of these algorithms is: a sensor being in the transmission range of another sensor defines a proximity constraint between both sensors, which can be exploited for localization. Bulusu et al. [86] and Niculescu et al. [87] developed distributed connectivity-based localization algorithms; Shang et al. [85] and Doherty et al. [88] developed centralized connectivitybased localization algorithms.

In [86], Bulusu et al. defined a connectivity metric, which is the ratio of the number of transmitter signals successfully received to the total number of signals from that transmitter, to measure the quality of communication for a specific transmitter-receiver pair. A receiver at an unknown location uses the centroid of its reference points as its location estimate, where a reference point is a transmitter with a known location and whose connectivity metric exceeds a certain threshold (90\% in [86]). An experiment was conducted in a $10 m \times 10 m$ outdoor parking lot using four reference points placed at the four corners of the $10 \mathrm{~m} \times 10 \mathrm{~m}$ square. The $10 \mathrm{~m} \times 10 \mathrm{~m}$ square was subdivided into 100 smaller $1 \mathrm{~m} \times 1 \mathrm{~m}$ grids and the receivers were placed at the grid points. Experimental results 
showed that for over $90 \%$ of the data points the localization error falls within $30 \%$ of the separation distance between two adjacent reference points.

The "DV(distance vector)-hop" approach developed by Niculescu et al. [87] starts with all anchors flooding their locations to other nodes in the network. The messages are propagated hop-by-hop and there is a hop-count in the message. Each node maintains an anchor information table and counts the least number of hops that it is away from an anchor. When an anchor receives a message from another anchor, it estimates the average distance of one hop using the locations of both anchors and the hop-count, and sends it back to the network as a correction factor. When receiving the correction factor, a non-anchor node is able to estimate its distance to anchors and performs trilateration to estimate its location. The algorithm was tested using simulation with a total of 100 nodes uniformly distributed in a circular region of diameter 10 . The average node degree, i.e., average number of neighbors per node, is 7.6. Simulation results showed that the algorithm has a mean error of $45 \%$ transmission range with $10 \%$ anchors; and has a reduced mean error of about $30 \%$ transmission range when the percentage of anchors increases above $20 \%$.

Shang et al. [85] developed a centralized algorithm by using multi-dimensional scaling (MDS). MDS was originally used in psychometrics and psychophysics and it is a set of data analysis techniques that displays the structure of distance-like data as a geometric picture. In their algorithm, the shortest paths (i.e., the number of hops) between all pairs of nodes are first computed, which are used to construct a distance matrix for MDS. Then MDS is applied to the distance matrix and an approximate value of the relative coordinates of each node is obtained. Finally, the relative coordinates are transformed to the absolute coordinates by aligning the estimated relative coordinates of anchors with their absolute coordinates. The location estimates obtained using earlier steps can be refined using a least-squares minimization. Simulation was conducted using 100 nodes uniformly distributed in a square of size $10 \times 10$ and four anchors were randomly placed in the region. The average node degree is 10 . Simulation results showed a localization error of 0.35 . Shang et al. further improved their algorithm in [89] by dividing the entire sensor network into overlapping local regions. Localization is performed in individual regions using the earlier described procedures. Then these local maps are patched together to form a global map by using common nodes shared between adjacent regions. The improved algorithm can achieve better performance on irregularly-shaped networks by avoiding the use of distance information between far away nodes. The improved algorithm can also be implemented in a distributed fashion.

In the centralized algorithm of Doherty et al. [88], the connectivity-based localization problem is formulated as a convex optimization problem and solved using existing algorithms for solving linear programs and semidefinite programming (SDP) algorithms. Semidefinite programs are a generalization of the linear programs and have the form:

$$
\begin{aligned}
\text { Minimize } & \mathbf{c}^{T} \mathbf{x} \\
\text { Subject to: } & \mathbf{F}(x)=\mathbf{F}_{0}+\mathbf{x}_{1} \mathbf{F}_{1}+\cdots+\mathbf{x}_{n} \mathbf{F}_{n} \\
& \mathbf{A x}<\mathbf{b} \\
& \mathbf{F}_{i}=\mathbf{F}_{i}^{T}
\end{aligned}
$$

where $\mathbf{x}=\left[\mathbf{x}_{1}, \mathbf{x}_{2}, \ldots, \mathbf{x}_{n}\right]^{T}$ and $\mathbf{x}_{i}$ represents the coordinate vector of node $i$, i.e., $\mathbf{x}_{i}=\left[x_{i}, y_{i}\right]$. The quantities $\mathbf{A}, \mathbf{b}$, $\mathbf{c}$ and $\mathbf{F}_{i}$ are all known. The inequality 39 is known as a linear matrix inequality. A connection between node $i$ and $j$ can be represented by a "radial constraint" on the node locations: $\left\|\mathbf{x}_{i}-\mathbf{x}_{j}\right\| \leq R$, where $R$ is the transmission range. This constraint is a convex constraint and can be transformed into a LMI using Schur complements [88]. A solution to the coordinates of the non-anchor nodes satisfying the radial constraints can be obtained by leaving the objective function $\mathbf{c}^{T} \mathbf{x}$ blank and solving the problem. Because there may be many possible coordinates of the non-anchor nodes satisfying the constraints, the solution may not be unique. If we set the element of $\mathbf{c}$ corresponding to $x_{i}$ (or $y_{i}$ ) to be 1 (or -1) and all other elements of $\mathbf{c}$ to be zero, the problem becomes a constrained maximization (or minimization) problem. A lower bound or an upper bound on $x_{i}$ (or $y_{i}$ ) satisfying the radial constraints can be computed, from which a rectangular box bounding the location estimates of the non-anchor nodes can be obtained. The algorithm was tested using simulation with a total of 200 nodes randomly placed in a square of size $10 R \times 10 R$ and the average node degree is 5.7 [88]. Simulation results showed that the mean location error is a monotonically decreasing function of the number of anchors. When the number of anchors is small, the estimated location is as poor as a random guess of the node's coordinates. The mean location error reduces to $R$ when the number of anchors increases to 18 ; it reduces to $0.5 R$ when the number of anchors increases to 50 .

In comparison with other localization algorithms, the most attractive feature of the connectivity-based localization algorithms is their simplicity. However they can only provide a coarse grained estimate of each node's location, which means that they are only suitable for applications requiring an approximate location estimate only. Also the localization error is highly dependent on the node density of the network, the number of anchors and the network topology. The location error will be larger in a network with a smaller node density, fewer anchors, or irregular network topology.

\section{Distance-Based Multihop Localization ALGORITHMS}

The core of distance-based localization algorithms is the use of inter-sensor distance measurements in a sensor network to locate the entire network. Based on the approach of processing the individual inter-sensor distance data, distance-based localization algorithms can be considered in two main classes: centralized algorithms and distributed algorithms. Centralized algorithms use a single central processor to collect all the individual inter-sensor distance data and produce a map of the entire sensor network, while distributed algorithms rely 
on self-localization of each node in the sensor network using the distances the node measures and the local information it collects from its neighbors. Next we review the main characteristics as well as relevant studies in the literature for each of the two classes and compare them at the end of the section.

\section{A. Centralized algorithms}

In certain networks where a centralized information architecture already exists, such as road traffic monitoring and control, environmental monitoring, health monitoring, and precision agriculture monitoring networks, the measurement data of all the nodes in the network are collected in a central processor unit. In such a network, it is convenient to use a centralized localization scheme.

Once feasible to implement, the main motive behind the interest in centralized localization schemes is the likelihood of providing more accurate location estimates than those provided by distributed algorithms. In the literature, there exist three main approaches for designing centralized distance-based localization algorithms: multidimensional scaling (MDS), linear programming and stochastic optimization approaches.

The MDS approach used in the connectivity-based localization algorithms mentioned in Section V, e.g., [85], can be readily extended to incorporate distance measurements into the corresponding optimization problem. Such an extension of the algorithm in [85] using the MDS approach can be found in [90]. In this work, the whole sensor network is divided into smaller groups where adjacent groups may share common sensors. Each group contains at least three anchors or sensors whose locations have already been estimated. MDS is used to estimate the relative locations of sensors in each group and build local maps. Local maps are then stitched together to form an estimated global map of the network by utilizing common sensors between adjacent local maps. The estimated locations of the anchors in this estimated global map are later iteratively aligned with the true locations of anchors to obtain the final estimated global map. Although this algorithm appears to have a distributed architecture, since a large number of iterations (implies a high communication cost) are required for the algorithm to converge, it is more appropriate to be implemented using a centralized architecture. Ji's algorithm was tested using a total of 400 nodes (10\% anchors) uniformly distributed in a square of $100 \times 100$ and a transmission range of $R=10$. The distance measurement error was assumed to be uniformly distributed in the range $[0, \eta]$. It was shown that when $\eta$ is $0,0.05 R, 0.25 R$ and $0.5 R$, the localization error is $0.1 R, 0.15 R, 0.3 R$ and $0.45 R$ respectively.

Similarly to the MDS approach, the semi-definite programming (SDP) approach used for connectivity-based localization algorithms can also be extended to incorporate distance measurements [88]. In [91] the distance-based sensor network localization problem is formulated in a quadratic form and solved using SDP; and in [92] the result in [91] is improved using a gradient search procedure to fine-tune the initial solution obtained using SDP.

The stochastic optimization approach suggests an alternative formulation and solution of the distance-based localization problem using combinatorial optimization notions and tools. The main tool used in this approach is the simulated annealing (SA) technique [93], which is a generalization of the well known Monte Carlo method in combinatorial optimization. One particular property of the SA method is its robustness against converging to a false local minimum. In order to apply this tool to the problem of localizing a sensor network with $m$ anchor nodes numbered from 1 to $m$ and $n$ non-anchor nodes numbered from $m+1$ to $m+n$, the location estimation problem is reformulated in an optimization framework as minimization of the cost function

$$
J=\sum_{i=m+1}^{m+n} \sum_{j \in N_{i}}\left(\left\|\hat{x}_{i}-\hat{x}_{j}\right\|-\tilde{d}_{i j}\right)^{2}
$$

over $\left\{\hat{x}_{i} \mid m+1 \leq i \leq m+n\right\}$, where $N_{i}, \hat{x}_{i}$ and $\tilde{d}_{i j}$ denote, respectively, the neighborhood of node $i$, the estimate of the location $x_{i}$ of node $\mathrm{i}$, and the measured distance between nodes $\mathrm{i}$ and $\mathrm{j}$.

An algorithm to solve the above optimization problem using the SA method is provided in [93]. The performance of this algorithm is improved in [94] utilizing the information about the sensor locations hidden in the knowledge of whether a given pair of sensors are neighbors and mitigating a certain kind of localization error caused by flip ambiguity, a concept which is described in detail in Section VII. The effectiveness of the enhanced algorithm in [94] is also demonstrated via simulations where the relation between the actual value $d_{i j}$ and the measured value $\tilde{d}_{i j}$ of the distance between nodes $\mathrm{i}$ and $\mathrm{j}$ is assumed to be $\tilde{d}_{i j}=d_{i j}\left(1+0.1 \xi_{i j}\right)$, where $\xi_{i j}$ is a zero-mean Gaussian noise of unit variance. The simulations were performed in a sensor network of 200 nodes uniformly distributed in a square of size 10 by 10 . The results of these simulations were compared with the ones obtained using the SDP approach with gradient search improvement [92] in Fig. 9, where the location estimation error is normalized by the transmission range. As can be seen in the figure, the SA algorithm has better accuracy than the SDP algorithm with gradient search. This is an expected result of robustness of SA against convergence to false local minima; however it is worth noting that the computational cost of the SA approach is higher.

\section{B. Distributed Localization}

Similarly to the centralized ones, the distributed distancebased localization approaches can be obtained as an extension of the distributed connectivity-based localization algorithms in Section V to incorporate the available inter-sensor distance information. In [87], after developing the "DV-hop" algorithm described in Section V, a modified version of this algorithm which includes distance measurements into the localization process, the "DV-distance" algorithm, is presented as well. The main idea in the "DV-distance" algorithm as compared to the "DV-hop" algorithm is propagation of measured distance among neighboring nodes instead of hop count.

Two similar approaches are the two-stage localization scheme of Savarese et al. [95] and the four-stage algorithm of Savvides et al. [96]. In the first stage of the scheme in 


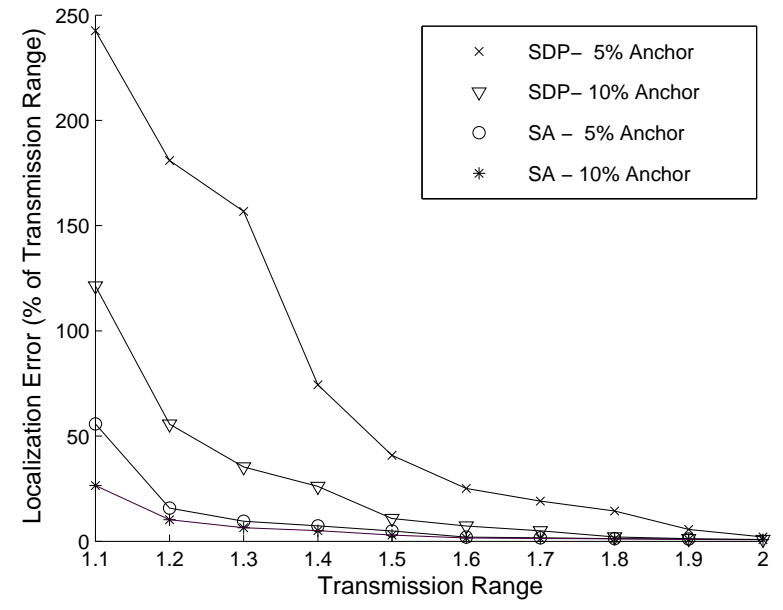

Fig. 9. Performance of SA algorithm with flip ambiguity mitigation and SDP algorithm with gradient search improvement.

[95], a "hop-terrain" algorithm, which is similar to the "DVhop" algorithm, is used to obtain an initial estimate of the node locations. In the second stage, the measured distances between neighboring nodes are used to refine the initial location estimates iteratively. At each iteration step, each node updates its location estimate by a least-squares trilateration using the location estimates of its neighbors and the measured distances. To mitigate location estimate errors caused by error propagation and unfavorable network topologies, a confidence value is assigned to each node's location, where an anchor has a higher confidence value (close to 1) and a non-anchor node with few neighbors and poor constellation has lower confidence value (close to 0 ). The proposed algorithm is tested via simulation as well in [95] using a sample network with 400 nodes, $5 \%$ of which are anchor nodes, uniformly placed in a 100 by 100 square and an average node degree greater than 7 . The simulation results demonstrated that the algorithm is able to achieve an average location estimation error of less than $33 \%$ of the transmission range in the presence of $5 \%$ distance measurement error (normalized by the transmission range).

The four-stage scheme of [96] is based on introduction of the notion of "tentative uniqueness", where a node is called "tentatively uniquely" localizable if it has at least three neighbors that are either non-collinear anchors or "tentatively uniquely" localizable nodes. In the first stage, the "tentatively uniquely" localizable nodes are selected. The locations of these tentatively uniquely localizable nodes are estimated in stages two and three. In the second stage, each non-anchor node produces its estimated distances to at least three anchors using a "DV-distance" like algorithm. An estimated distance to an anchor node allows the location of the non-anchor node to be constrained inside a square centered at that anchor node. In comparison with a circle, the use of a square may simplify computation. The estimated distances to more than three anchors allow the location of the non-anchor node to be confined inside a rectangular box, which is the intersection of the squares corresponding to each of these anchors. The location of the non-anchor node is estimated to be at the center of the rectangular box. The initial location estimates obtained in the second stage are refined in the third stage by a least-squares trilateration using the location estimates of the neighboring nodes and the measured distances. In the final stage of the algorithm, the location of each node deemed not tentatively uniquely localizable in stage one is estimated using the location estimates of its tentatively uniquely localizable neighbors.

All of the above three algorithms [87], [95], [96] have three phases [97]: a) determination of the distances between nonanchor nodes and anchor nodes; b) derivation of the location of at least some non-anchor nodes from their distances to the anchors; c) refinement of the location estimates using measured distances between neighbors. In [97] the performances of the three algorithms and some variants of them were compared and it was concluded that the algorithms have comparable performance and which algorithm has better accuracy depends on the specific application conditions such as distance measurement error, vertex degree and percentage of anchors. The algorithm proposed by Nagpal et al. [98] more recently can be classified into the same category as the above three algorithms.

There exists another category of distributed localization algorithms in the literature, where local maps are constructed using distance measurements between neighboring nodes first and then common nodes between local maps are used to stitch them together to form a global map. The localization algorithms by Ji et al. [90] and Čapkun et al. [99] are typical examples of this category. In the algorithm of Capkun et al. [99], each node builds its local coordinate system and the locations of its neighbors are calculated in the local coordinate system. Then the directions of the local coordinate systems are aligned to be the same using common nodes between adjacent local coordinate systems. Finally, the local coordinate systems are reconciled into a global coordinate system using linear translation. Error propagation and the large number of iterations required for the algorithm to converge are the major problems in these algorithms.

A recent direction of research in distributed sensor network localization is the use of particle filters [100]. Particle filters have been used in navigation and tracking [101]. In [102], Ihler et al. formulated the sensor network localization problem as an inference problem on a graphical model and applied a variant of belief propagation (BP) techniques, the so-called nonparametric belief propagation (NBP) algorithm, to obtain an approximate solution to the sensor locations. In [102], the NBP idea is implemented as an iterative local message exchange algorithm, in each step of which each sensor node quantifies its "belief" about its location estimate, sends this belief information to its neighbors, receives relevant messages from them, and then iteratively updates its belief. The iteration process is terminated only when some convergence criterion is met about the beliefs and location estimates of the sensors in the network. The main advantages of the NBP algorithm are its easy implementation in a distributed fashion and sufficiency of a small number of iterations to converge. Furthermore it is capable of providing information about location estimation uncertainties and accommodating non-Gaussian distance 
measurement errors. It is demonstrated via simulations [102] that the overall performance of NBP is comparable to that of a centralized MAP (maximum a posteriori) estimate. Some future research directions to further improve the NBP approach can be found in [102].

\section{Centralized versus Distributed Algorithms}

Centralized and distributed distance-based localization algorithms can be compared from perspectives of location estimation accuracy, implementation and computation issues, and energy consumption. It is worth noting that decentralized localization is strictly harder than centralized, i.e., any algorithm for decentralized localization can always be applied to centralized problems, but not the reverse.

From the perspective of location estimation accuracy, centralized algorithms are likely to provide more accurate location estimates than distributed algorithms. However centralized algorithms suffer from the scalability problem and generally are not feasible to be implemented for large scale sensor networks. Other disadvantages of centralized algorithms, as compared to distributed algorithms, are their requirement of higher computational complexity and lower reliability due to accumulated information inaccuracies/losses caused by multihop transmission over a wireless network.

On the other hand, distributed algorithms are more difficult to design because of the potentially complicated relationship between local behavior and global behavior, e.g., algorithms that are locally optimal may not perform well in a global sense. Optimal distribution of the computation of a centralized algorithm in a distributed implementation in general is an unsolved research problem. Error propagation is another potential problem in distributed algorithms. Moreover, distributed algorithms generally require multiple iterations to arrive a stable solution which may cause the localization process to take longer time than the acceptable in some cases.

To compare the centralized and distributed distance-based localization algorithms from the communication energy consumption perspective, one needs to consider the individual amounts of energy required for each type of operation in the localization algorithm in the specific hardware and the transmission range setting. Depending on the setting, the energy required for transmitting a single bit could be used to execute 1,000 to 2,000 instructions [103]. Centralized algorithms in large networks require each sensor's measurements to be sent over multiple hops to a central processor, while distributed algorithms require only local information exchange between neighboring nodes but many such local exchanges may be required, depending on the number of iterations needed to arrive at a stable solution. A comparison of the communication energy efficiencies of centralized and distributed algorithms can be found in [104]. It was concluded in [104] that in general, if in a given sensor network and distributed algorithm, the average number of hops to the central processor exceeds the necessary number of iterations, then the distributed algorithm will be more energy-efficient than a typical centralized algorithm.

\section{Graph Theoretic Research Problems in Distance-BASED SENSOR NeTwork LOCALIZATION}

Despite a significant number of approaches developed for WSN localization, there are still many unsolved problems in the area. The challenges to be addressed are both in analytical characterization of the sensor networks (from the aspect of localization) and development of (efficient) localization algorithms for various classes of sensor networks under a variety of conditions. In this section, we present some of these research problems with a discussion on possible approaches to them. Although these problems may also exist in localization using other types of measurement techniques (e.g., TDOA and AOA), we focus on distance-based sensor network localization.

A fundamental problem in distance-based sensor network localization is whether a given sensor network is uniquely localizable or not. A framework that is useful for analyzing and solving the problem is graph theory [105]-[109]. In a graph theoretical framework, a sensor network can be represented by a graph $G=(V, E)$ with a vertex set $V$ and an edge set $E$, where each vertex $i \in V$ is associated with a sensor node $s_{i}$ in the network, and each edge $(i, j) \in E$ corresponds to a sensor pair $s_{i}, s_{j}$ for which the inter-sensor distance $d_{i j}$ is known. The location information about the sensors corresponds to a representation of the representative graph. In general, a $d$ dimensional $(d \in\{2,3\})$ representation of a graph $G=(V, E)$ is a mapping $\bar{p}: V \rightarrow \Re^{d}$, which assigns a location in $\Re^{d}$ to each vertex in $V$. Given a graph $G=(V, E)$ and a $d$ dimensional representation of it, the pair $(G, \bar{p})$ is called a $d$-dimensional framework.

A particular graph property associated with unique localizability of sensor networks is global rigidity [106], [108], [109]. A framework $(G, \bar{p})$ is globally rigid if every framework $\left(G, \bar{p}_{1}\right)$ satisfying $\|\bar{p}(i)-\bar{p}(j)\|=\left\|\bar{p}_{1}(i)-\bar{p}_{1}(j)\right\|$ for any vertex pair $i, j \in V$, which are connected by an edge in $E$, also satisfies the same equality for any other vertex pairs that are not connected by a single edge. A relaxed form of global rigidity is rigidity: A framework $(G, \bar{p})$ is rigid if there exists a sufficiently small positive constant $\varepsilon$ such that every framework $\left(G, \bar{p}_{1}\right)$ satisfying (i) $\left\|\bar{p}(i)-\bar{p}_{1}(i)\right\|<\varepsilon$ for all $i \in V$ and (ii) $\|\bar{p}(i)-\bar{p}(j)\|=\left\|\bar{p}_{1}(i)-\bar{p}_{1}(j)\right\|$ for any vertex pair $i, j \in V$, which are connected by an edge in $E$, also satisfies the equality in (ii) for any other vertex pairs that are not connected by a single edge.

If a framework $(G, \bar{p})$ is rigid but not globally rigid, like the ones in Fig. 10, there exist two types of discontinuous deformations that can prevent a representation of $G$ consistent with $\bar{p}$, i.e., a representation $\left(G, \bar{p}_{1}\right)$ satisfying $\| \bar{p}(i)-$ $\bar{p}(j) \quad\|=\| \bar{p}_{1}(i)-\bar{p}_{1}(j) \|$ for any vertex pair $i, j \in V$, which are connected by an edge in $E$, from being unique (in the sense that it differs from other such representations at most by translation, rotation or reflection) [107], [110]: flip and discontinuous flex ambiguities. In flip ambiguities in $\Re^{d},(d \in\{2,3\})$, at least a vertex (sensor node) $v$ has a set of neighbors which span a $(d-1)$-dimensional subspace, which leads to the possibility of the neighbors forming a mirror through which $v$ can be reflected. Fig. 10(a) depicts an 
example of flip ambiguity. In discontinuous flex ambiguities, the temporary removal of an edge or, in some cases, a set of edges allows the remaining part of the graph to be flexed to a different realization (which cannot be obtained from the original realization by translation, rotation or reflection) and the removed edge reinserted with the same length. Fig. 10(b) depicts an example.

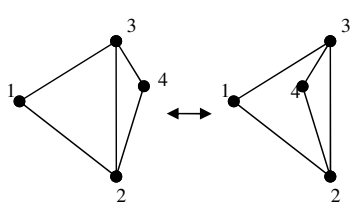

(a)

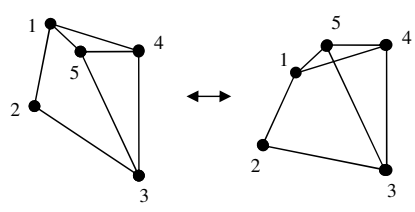

(b)
Fig. 10. An illustration of discontinuous deformations on non-globally rigid frameworks: (a) Flip ambiguity: vertex 4 can be reflected across the edge $(2,3)$ to a new location without violating the distance constraints. (b) Discontinuous flex ambiguity: removing the edge $(1,4)$, flexing the edge triple $(1,5),(1,2)$, $(2,3)$, and reinserting the edge $(1,4)$ so that the distance constraints are not violated in the end, we obtain a new new framework.

Use of graph rigidity and global rigidity notions in sensor network localization are well described and their importance is well demonstrated from both the algorithmic and the analytic aspects in the recent literature [98], [106], [108], [111]. Particularly, it is established in [105], [106] that a necessary and sufficient condition for unique localization of a $d$-dimensional sensor network is global rigidity of any $d$-dimensional representation $(G, \bar{p})$, where $G$ is the representative graph of the sensor network and the edge lengths $\|\bar{p}(i)-\bar{p}(j)\|$ imposed by $\bar{p}$ are equal to the corresponding known inter-sensor distances $d_{i j}$, assuming that the absolute positions of at least three sensors in $\Re^{2}$ (which do not lie on the same line) or four sensors in $R^{3}$ (which do not lie on the same plane) are known. Formal statement of this relation can be found in [105], [106].

Note that the necessity of global rigidity for unique localization as stated is valid for general situations where other a priori information is not helpful. Rigidity is needed, in any case, to have a finite number of solutions. However, in some cases where $(G, \bar{p})$ is rigid but not globally rigid, some additional a priori information may compensate the need for global rigidity. For example, consider a sensor network that can be represented by a unit disk graph, where there is an edge between representative vertices of two sensor nodes if and only if the distance between them is less than a certain threshold $R>0$, which is called the transmission range or sensing radius [106]. Then the ambiguities due to the non-globally rigid nature of the representative graph may sometimes be eliminated using the unit disk graph properties as demonstrated in Fig. 11. In practice, a wireless sensor always has a limited transmission range, which implies that a WSN may have the property of a unit disk graph. Therefore global rigidity is only a sufficient condition for unique localization of a WSN; the necessary condition for unique localization is still an open research problem.

A more challenging research problem is analyzing the characteristics of wireless sensor networks (from the aspect of localization) in the case of noisy distance measurements.

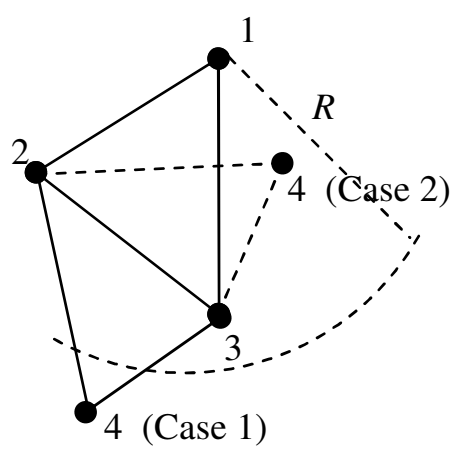

Fig. 11. Localization of a non-globally rigid unit-disk framework in $\Re^{2}$ : assume that the location of vertices $1,2,3$ and the lengths of the edges $(2,4)$ and $(3,4)$ are known and that there is no edge between 1 and 4 . There exist two possible locations for vertex 4 in general: Case 1 and Case 2 . We can eliminate Case 2 using the unit-disk property since for this case there had to be an edge between vertices 1 and 4 since $d_{14}<R$. Hence Case 1 gives the correct unique location of vertex 4 .

We have little knowledge in this area. For example, it is a common knowledge that in the presence of noisy distance measurements, a node (in $\Re^{2}$ ) is likely to have flip ambiguity problem if its neighbors are nearly collinear. However there is little work in quantifying this relationship. A recent work focusing on robust distributed localization of sensor networks with certain distance measurement errors and ambiguities caused by these errors is presented in [107]. In this paper, certain criteria are provided in selection of the subgraphs of the representative graph of a network to be used in a localization algorithm robust against such errors. The analysis in [107], however, is not complete and there may be other criteria that may better characterize robustness of a given sub-network against distance measurement errors.

Another relevant research problem is understanding and utilizing the error propagation characteristics in a sensor network. This issue emerges especially in estimation of the location of non-immediate neighbors of anchor sensors, i.e., $k$-hop neighbors of anchor nodes with $k \geq 2$. Other things being equal, a node further away from anchor nodes is likely to have a larger location estimation error, because its location estimation error is not only affected by the distance measurement errors to its neighbors but also affected by the location estimation errors of its neighbors using which the node's location is estimated. Numerous simulations and experimental studies suggested that in addition to distance measurement error, error propagation (as well as location estimation error) may be affected by node degree, network topology, and the distribution of both non-anchor and anchor nodes. Some of the related tools can be seen in [102]. Other relevant work includes the papers by Niculescu et al. [112] and by Savvides et al. [113]. In [112], Niculescu et al. used a combination of the Cramér-Rao bound and simulations to investigate the error characteristics of four classes of multihop APS (Ad Hoc positioning systems) algorithms. In [113] Savvides et al. also used a combination of the Cramér-Rao bound and simulations to investigate the error characteristics for a specific scenario 
in which anchors are located near the boundary of the region and non-anchor nodes are located inside the region. Some qualitative trends on how localization error varies with average node degree, number of anchors and distance to anchors are observed. A potential problem with using the Cramér-Rao bound to study the performance of a localization algorithm is that the Cramér-Rao bound assumes the underlying estimator is unbiased. This assumption needs to be validated with the estimators used in various localization algorithms, and in particular, the class of algorithms which minimize the sum of the square of the difference between measured distances and estimated distances. The aforementioned flip ambiguity and discontinuous flex ambiguity problems make such validation particularly difficult. An estimator may produce an unbiased estimate in one topology (e.g., a dense network with high node degree) but give a biased estimate in another topology (e.g., a spare network with low node degree). We are yet to develop more comprehensive knowledge in this large and fascinating area.

Still another research area is concerned with reducing the computational complexity of location algorithms. It is concluded in the literature that the computational complexity of an arbitrary location procedure is, in general, exponential in the number of sensor nodes, unless an iterative procedure is applied to sensor networks with certain classes of representative graphs such as trilateration and quadrilateration graphs [105], [106], [108]. Since trilateration and quadrilateration representative graphs provide proven reduced computational complexity in localization and actually there are systematic methods to locate networks with such representative graphs, it is of interest to develop mechanisms to make these methods applicable for certain other classes of representative graphs as well.

Finally, we note current interest in characterizing statistical properties of random sensor networks which will ensure, at least with high probability, that the network is localizable, or even possesses trilateration structure, so that localization computations are straightforward and indeed decentralizable [105], [108], [114].

\section{SUMMARY}

Wireless sensor network localization has attracted significant research interest. This interest is expected to grow further with the proliferation of wireless sensor network applications. This paper has provided a review of the measurement techniques in WSN localization and the corresponding localization algorithms. These localization algorithms were divided into one-hop localization algorithms and multi-hop localization algorithms. A detailed investigation on connectivity-based and distance-based localization algorithms were presented because of their popularity in wireless sensor network localization. Despite significant research developments in the area, there are still quite many unsolved problems in wireless sensor network localization. A discussion on some fundamental research problems in distance-based location and possible approaches to these problem was also presented in this paper.

\section{ACKNOWLEDGMENT}

The authors would like to thank the anonymous reviewers for their helpful comments which have significantly improved the quality of the paper.

\section{REFERENCES}

[1] C.-Y. Chong and S. Kumar, "Sensor networks: evolution, opportunities, and challenges," Proceedings of the IEEE, vol. 91, no. 8, pp. 12471256, 2003.

[2] S. Guolin, C. Jie, G. Wei, and K. J. R. Liu, "Signal processing techniques in network-aided positioning: a survey of state-of-the-art positioning designs," IEEE Signal Processing Magazine, vol. 22, no. 4, pp. 12-23, 2005.

[3] A. H. Sayed, A. Tarighat, and N. Khajehnouri, "Network-based wireless location: challenges faced in developing techniques for accurate wireless location information," IEEE Signal Processing Magazine, vol. 22, no. 4, pp. 24-40, 2005.

[4] F. Gustafsson and F. Gunnarsson, "Mobile positioning using wireless networks: possibilities and fundamental limitations based on available wireless network measurements," IEEE Signal Processing Magazine, vol. 22, no. 4, pp. 41-53, 2005.

[5] N. Patwari, J. Ash, S. Kyperountas, I. Hero, A.O., R. Moses, and N. Correal, "Locating the nodes: cooperative localization in wireless sensor networks," IEEE Signal Processing Magazine, vol. 22, no. 4, pp. 54-69, 2005.

[6] D. Koks, "Numerical calculations for passive geolocation scenarios, Tech. Rep. DSTO-RR-0000, 2005.

[7] T. Rappaport, J. Reed, and B. Woerner, "Position location using wireless communications on highways of the future," IEEE Communications Magazine, vol. 34, no. 10, pp. 33-41, 1996.

[8] T. E. Biedka, J. H. Reed, and B. D. Woerner, "Direction finding methods for CDMA systems," in Thirteenth Asilomar Conference on Signals, Systems and Computers, vol. 1, 1996, pp. 637-641.

[9] D. W. Bliss and K. W. Forsythe, "Angle of arrival estimation in the presence of multiple access interference for CDMA cellular phone systems," in Proceedings of the 2000 IEEE Sensor Array and Multichannel Signal Processing Workshop, 2000, pp. 408-412.

[10] I. Ziskind and M. Wax, "Maximum likelihood localization of multiple sources by alternating projection," IEEE Transactions on Acoustics, Speech, and Signal Processing, vol. 36, no. 10, pp. 1553-1560, 1988.

[11] L. Jian, B. Halder, P. Stoica, and M. Viberg, "Computationally efficient angle estimation for signals with known waveforms," IEEE Transactions on Signal Processing, vol. 43, no. 9, pp. 2154-2163, 1995.

[12] B. Halder, M. Viberg, and T. Kailath, "An efficient non-iterative method for estimating the angles of arrival of known signals," in The TwentySeventh Asilomar Conference on Signals, Systems and Computers, 1993, pp. 1396-1400.

[13] B. G. Agee, "Copy/DF approaches for signal specific emitter location," in Conference Record of the Twenty-Fifth Asilomar Conference on Signals, Systems and Computers, vol. 2, 1991, pp. 994-999.

[14] R. Schmidt, "Multiple emitter location and signal parameter estimation," IEEE Transactions on Antennas and Propagation, vol. 34, no. 3 , pp. 276-280, 1986.

[15] R. Roy and T. Kailath, "ESPRIT-estimation of signal parameters via rotational invariance techniques," IEEE Transactions on Acoustics, Speech, and Signal Processing, vol. 37, no. 7, pp. 984-995, 1989.

[16] A. Paulraj, R. Roy, and T. Kailath, "A subspace rotation approach to signal parameter estimation," Proceedings of the IEEE, vol. 74, no. 7, pp. 1044-1046, 1986.

[17] N. Tayem and H. M. Kwon, "Conjugate ESPRIT (C-SPRIT)," IEEE Transactions on Antennas and Propagation, vol. 52, no. 10, pp. 2618 2624, 2004.

[18] A. Barabell, "Improving the resolution performance of eigenstructurebased direction-finding algorithms," in IEEE International Conference on Acoustics, Speech, and Signal Processing, vol. 8, 1983, pp. 336339.

[19] M. Kaveh and A. Bassias, "Threshold extension based on a new paradigm for MUSIC-type estimation," in International Conference on Acoustics, Speech, and Signal Processing, vol. 5, 1990, pp. 2535-2538.

[20] R. KUMARESAN and D. W. TUFTS, "Estimating the angles of arrival of multiple plane waves," IEEE Transactions on Aerospace and Electronic Systems, vol. AES-19, pp. 134-139, 1983. 
[21] J. W. Pierre and M. Kaveh, "Experimental evaluation of higherresolution direction-finding algorithms using a calibrated sensor array testbed," Digital Signal Processing, vol. 5, pp. 243-254, 1995.

[22] R. Klukas and M. Fattouche, "Line-of-sight angle of arrival estimation in the outdoor multipath environment," IEEE Transactions on Vehicular Technology, vol. 47, no. 1, pp. 342-351, 1998.

[23] S. V. Schell and W. A. Gardner, "High-resolution direction finding," Handbook of Statistics, vol. 10, pp. 755-817, 1993.

[24] K. Romer, "The lighthouse location system for smart dust," in Proceedings of MobiSys 2003 (ACM/USENIX Conference on Mobile Systems, Applications, and Services), 2003, pp. 15-30.

[25] D. McCrady, L. Doyle, H. Forstrom, T. Dempsey, and M. Martorana, "Mobile ranging using low-accuracy clocks," IEEE Transactions on Microwave Theory and Techniques, vol. 48, no. 6, pp. 951-958, 2000.

[26] G. Carter, Coherence and Time Delay Estimation. Piscataway, NJ: IEEE Press, 1993.

[27] C. Knapp and G. Carter, "The generalized correlation method for estimation oftime delay," IEEE Transansaction on Acoustics, Speech, Signal Processing, vol. 24, no. 4, p. 320327, 1976.

[28] N. B. Priyantha, A. Chakraborty, and H. Balakrishnan, "The cricket location-support system," in Proc. of the Sixth Annual ACM International Conference on Mobile Computing and Networking, 2000, pp. $32-43$.

[29] J.-Y. Lee and R. Scholtz, "Ranging in a dense multipath environment using an UWB radio link," IEEE Journal on Selected Areas in Communications, vol. 20, no. 9, pp. 1677-1683, 2002.

[30] S. Gezici, Z. Tian, G. Giannakis, H. Kobayashi, A. Molisch, H. Poor, and Z. Sahinoglu, "Localization via ultra-wideband radios: a look at positioning aspects for future sensor networks," IEEE Signal Processing Magazine, vol. 22, no. 4, pp. 70-84, 2005.

[31] G. Carter, "Time delay estimation for passive sonar signal processing," IEEE Transactions on Acoustics, Speech, and Signal Processing, vol. 29 , no. 3, pp. 463-470, 1981.

[32] W. A. Gardner and C. K. Chen, "Signal-selective time-difference-ofarrival estimation for passive location of man-made signal sources in highly corruptive environments. i. theory and method," IEEE Transactions on Signal Processing, vol. 40, no. 5, pp. 1168-1184, 1992.

[33] C. K. Chen and W. A. Gardner, "Signal-selective time-difference of arrival estimation for passive location of man-made signal sources in highly corruptive environments. ii. algorithms and performance," IEEE Transactions on Signal Processing, vol. 40, no. 5, pp. 1185-1197, 1992.

[34] P. Bergamo and G. Mazzini, "Localization in sensor networks with fading and mobility," in The 13th IEEE International Symposium on Personal, Indoor and Mobile Radio Communications, vol. 2, 2002, pp. 750-754.

[35] E. Elnahrawy, X. Li, and R. Martin, "The limits of localization using signal strength: a comparative study," in First Annual IEEE Conference on Sensor and Ad-hoc Communications and Networks, 2004, pp. 406414.

[36] D. Madigan, E. Einahrawy, R. Martin, W.-H. Ju, P. Krishnan, and A. Krishnakumar, "Bayesian indoor positioning systems," in IEEE INFOCOM 2005, vol. 2, 2005, pp. 1217-1227.

[37] D. Niculescu and B. Nath, "Localized positioning in ad hoc networks," in IEEE International Workshop on Sensor Network Protocols and Applications, 2003, pp. 42-50.

[38] N. Patwari, A. Hero, M. Perkins, N. Correal, and R. O'Dea, "Relative location estimation in wireless sensor networks," IEEE Transactions on Signal Processing, vol. 51, no. 8, pp. 2137-2148, 2003.

[39] T. S. Rappaport, Wireless Communications: Principles and Practice, 2nd ed. Prentice Hall PTR, 2001.

[40] D. C. Cox, R. Murray, and A. Norris, " $800 \mathrm{mhz}$ attenuation measured in and around suburban houses," $A T \& T$ Bell Laboratory Technical Journal, vol. 673, no. 6, pp. 921-954, 1984.

[41] R. Bernhardt, "Macroscopic diversity in frequency reuse radio systems," IEEE Journal on Selected Areas in Communications, vol. 5, no. 5, pp. 862-870, 1987.

[42] P. Bahl and V. Padmanabhan, "RADAR: an in-building RF-based user location and tracking system," in IEEE INFOCOM, vol. 2, 2000, pp. 775-784.

[43] P. Prasithsangaree, P. Krishnamurthy, and P. Chrysanthis, "On indoor position location with wireless LANs," in The 13th IEEE International Symposium on Personal, Indoor and Mobile Radio Communications, vol. 2, 2002, pp. 720-724.

[44] P. Krishnan, A. Krishnakumar, W.-H. Ju, C. Mallows, and S. Gamt, "A system for LEASE: location estimation assisted by stationary emitters for indoor RF wireless networks," in IEEE INFOCOM, vol. 2, 2004, pp. 1001-1011.
[45] T. Roos, P. Myllymaki, and H. Tirri, "A statistical modeling approach to location estimation," IEEE Transactions on Mobile Computing, vol. 1, no. 1, pp. 59-69, 2002.

[46] S. Ray, W. Lai, and I. Paschalidis, "Deployment optimization of sensornet-based stochastic location-detection systems," in IEEE INFOCOM 2005, vol. 4, 2005, pp. 2279-2289.

[47] D. J. Torrieri, "Statistical theory of passive location systems," IEEE Transactions on Aerospace and Electronic Systems, vol. AES-20, no. 2, pp. 183-198, 1984.

[48] M. Gavish and A. J. Weiss, "Performance analysis of bearing-only target location algorithms," IEEE Transactions on Aerospace and Electronic Systems, vol. 28, no. 3, pp. 817-828, 1992.

[49] R. G. Stanfield, "Statistical theory of DF finding," Journal of IEE, vol. 94 , no. 5 , pp. $762-770,1947$

[50] C. J. Ancker, "Airborne direction finding - theory of navigation errors," IRE Transactions on Aeronautical and Navigational Electronics, vol. 5 , pp. 199-210, 1958.

[51] W. H. Foy, "Position-location solutions by Taylor-series estimation," IEEE Transaction on Aerospace and Electronic Systems, vol. AES-12, no. 2, pp. 187-194, 1976.

[52] J. L. Poirot and G. V. McWilliams, "Application of linear statistical models to radar location techniques," IEEE Transaction on Aerospace and Electronic Systems, vol. AES-10, pp. 830-834, 1974.

[53] S. Bancroft, "Algebraic solution of the GPS equations," IEEE Transactions on Aerospace and Electronic Systems, vol. AES-21, no. 1, pp. 56-59, 1985.

[54] B. T. Fang, "Simple solutions for hyperbolic and related position fixes," IEEE Transactions on Aerospace and Electronic Systems, vol. 26, no. 5, pp. 748-753, 1990.

[55] J. Smith and J. Abel, "The spherical interpolation method of source localization," IEEE Journal of Oceanic Engineering, vol. 12, no. 1, pp. 246-252, 1987.

[56] J. S. Abel, "A divide and conquer approach to least-squares estimation," IEEE Transactions on Aerospace and Electronic Systems, vol. 26, no. 2, pp. 423-427, 1990.

[57] Y. T. Chan and K. C. Ho, "A simple and efficient estimator for hyperbolic location," IEEE Transactions on Signal Processing, vol. 42, no. 8, pp. $1905-1915,1994$

[58] K. Dogancay, "Emitter localization using clustering-based bearing association," IEEE Transactions on Aerospace and Electronic Systems, vol. 41, no. 2, pp. 525-536, 2005.

[59] S. Drake and K. Dogancay, "Geolocation by time difference of arrival using hyperbolic asymptotes," in IEEE International Conference on Acoustics, Speech, and Signal Processing, vol. 2, 2004, pp. ii-361-4.

[60] G. M. Crippen and T. F. Havel, Distance Geometry and Molecular Conformation. New York: John Wiley and Sons Inc., 1988.

[61] L. M. Blumenthal, Theory and Applications Distance Geometry. Oxford University Press, 1953

[62] M. Cao, B. Anderson, and A. Morse, "Localization with imprecise distance information in sensor networks," in Proc. Joint IEEE Conf on Decision and Control and European Control Conf., 2005, pp. 28292834.

[63] M. Cao, A. Morse, and B. Anderson, "Sensor network localization with imprecise distances," Systems and Control Letters, vol. 55, pp. 887-893, 2006.

[64] A. N. Bishop, P. N. Pathirana, B. Fidan, B. D. Anderson, and G. Mao, "Passive angle measurement based localization consistency via geometric constraints," in submitted for publication in Information, Decision and Control 2007, 2006.

[65] R. Battiti, M. Brunato, and A. Villani, "Statistical learning theory for location fingerprinting in wireless LANs," Informatica e Telecomunicazioni, University of Trento, Tech. Rep. DIT-02-086, October 2002.

[66] L. M. Ni, L. Yunhao, L. Yiu Cho, and A. P. Patil, "LANDMARC: indoor location sensing using active rfid," in Proceedings of the First IEEE International Conference on Pervasive Computing and Communications (PerCom 2003)., 2003, pp. 407-415.

[67] P. Bahl, V. N. Padmanabhan, and A. Balachandran, "Enhancement to the RADAR user location and tracking system," Microsoft Research Technical Report MSR-TR-2000-12, February 2000.

[68] D. L. Hall, Mathematical Techniques in Multisensor Data Fusion. Artech House Publishers, 2004.

[69] M. McGuire, K. N. Plataniotis, and A. N. Venetsanopoulos, "Data fusion of power and time measurements for mobile terminal location," IEEE Transactions on Mobile Computing, vol. 4, no. 2, pp. 142-153, 2005, 1536-1233. 
[70] C. Li and W. Zhuang, "Hybrid TDOA/AOA mobile user location for wideband CDMA cellular systems," IEEE Transactions on Wireless Communications, vol. 1, no. 3, pp. 439-447, 2002.

[71] Z. Gu and E. Gunawan, "Radiolocation in CDMA cellular system based on joint angle and delay estimation," Wireless Personal Communications, vol. 23, no. 3, pp. $297-309,2002$.

[72] T. Kleine-Ostmann and A. E. Bell, "A data fusion architecture for enhanced position estimation in wireless networks," IEEE Communications Letters, vol. 5, no. 8, pp. 343-345, 2001.

[73] N. Thomas, D. Cruickshank, and D. Laurenson, "Performance of TDOA-AOA hybrid mobile location system," in Procedings International Conference on $3 G$ Mobile Communication Technologies, 2001, pp. 216-220.

[74] A. Catovic and Z. Sahinoglu, "The Cramér-Rao bounds of hybrid TOA/RSS and TDOA/RSS location estimation schemes," IEEE Communications Letters, vol. 8, no. 10, pp. 626-628, 2004.

[75] C. Li and W. Zhuang, "Nonline-of-sight error mitigation in mobile location," IEEE Transactions on Wireless Communications, vol. 4 no. 2 , pp. $560-573,2005$.

[76] X. Li, "A selective model to suppress NLOS signals in angle-ofarrival (AOA) location estimation," in The Ninth IEEE International Symposium on Personal, Indoor and Mobile Radio Communications, vol. 1, 1998, pp. 461-465.

[77] S.-S. Woo, H.-R. You, and J.-S. Koh, "The NLOS mitigation technique for position location using IS-95 CDMA networks," in 52nd Vehicular Technology Conference, IEEE VTS-Fall VTC 2000, vol. 6, 2000, pp. 2556-2560.

[78] S. Gezici, H. Kobayashi, and H. V. Poor, "Nonparametric nonline-ofsight identification," in IEEE 58th Vehicular Technology Conference, VTC 2003-Fall, vol. 4, 2003, pp. 2544-2548.

[79] S. Venkatraman, J. Caffery, J., and Y. Heung-Ryeol, "A novel TOA location algorithm using los range estimation for NLOS environments," IEEE Transactions on Vehicular Technology, vol. 53, no. 5, pp. 15151524, 2004, 0018-9545.

[80] P.-C. Chen, "A non-line-of-sight error mitigation algorithm in location estimation," in IEEE Wireless Communications and Networking Conference, 1999 , pp. 316-320.

[81] M. P. Wylie and J. Holtzman, "The non-line of sight problem in mobile location estimation," in 5th IEEE International Conference on Universal Personal Communications, vol. 2, 1996, pp. 827-831.

[82] L. Cong and W. Zhuang, "Non-line-of-sight error mitigation in TDOA mobile location," in IEEE Global Telecommunications Conference, GLOBECOM '01., vol. 1, 2001, pp. 680-684.

[83] M. P. Wylie-Green and S. S. Wang, "Robust range estimation in the presence of the non-line-of-sight error," in IEEE VTS 54th Vehicular Technology Conference, VTC 2001 Fall, vol. 1, 2001, pp. 101-105.

[84] X. Wang, Z. Wang, and B. O'Dea, "A TOA-based location algorithm reducing the errors due to non-line-of-sight (NLOS) propagation," IEEE Transactions on Vehicular Technology, vol. 52, no. 1, pp. 112116,2003

[85] Y. Shang, W. Ruml, Y. Zhang, and M. Fromherz, "Localization from connectivity in sensor networks," IEEE Transactions on Parallel and Distributed Systems, vol. 15, no. 11, pp. 961-974, 2004.

[86] N. Bulusu, J. Heidemann, and D. Estrin, "GPS-less low-cost outdoor localization for very small devices," IEEE Personal Communications, vol. 7, no. 5, pp. 28-34, 2000.

[87] D. Niculescu and B. Nath, "Ad hoc positioning system (APS)," in IEEE GLOBECOM, vol. 5, 2001, pp. 2926-2931.

[88] L. Doherty, K. pister, and L. El Ghaoui, "Convex position estimation in wireless sensor networks," in IEEE INFOCOM, vol. 3, 2001, pp. $1655-1663$.

[89] Y. Shang and W. Ruml, "Improved MDS-based localization," in IEEE INFOCOM 2004, vol. 4, 2004, pp. 2640-2651.

[90] X. Ji and H. Zha, "Sensor positioning in wireless ad-hoc sensor networks using multidimensional scaling," in IEEE INFOCOM, vol. 4 , 2004, pp. 2652-2661.

[91] P. Biswas and Y. Ye, "Semidefinite programming for ad hoc wireless sensor network localization," in Third International Symposium on Information Processing in Sensor Networks, 2004, pp. 46-54.

[92] T.-C. Liang, T.-C. Wang, and Y. Ye, "A gradient search method to round the semidefinite programming relaxation for ad hoc wireless sensor network localization," Standford University, Tech. Rep., August 262004.

[93] A. A. Kannan, G. Mao, and B. Vucetic, "Simulated annealing based localization in wireless sensor network," in The 30th IEEE Conference on Local Computer Networks, 2005, pp. 513-514.
[94] _ _ "Simulated annealing based wireless sensor network localization with flip ambiguity mitigation," in 63rd IEEE Vehicular Technology Conference, 2006, pp. 1022- 1026.

[95] C. Savarese and J. Rabaey, "Robust positioning algorithms for distributed ad-hoc wireless sensor networks," in Proceedings of the General Track: 2002 USENIX Annual Technical Conference, 2002, pp. 317-327.

[96] A. Savvides, H. Park, and M. B. Srivastava, "The bits and flops of the n-hop multilateration primitive for node localization problems," in International Workshopon Sensor Networks Application, 2002, pp. $112-121$.

[97] K. Langendoen and N. Reijers, "Distributed localization in wireless sensor networks: a quantative comparison," Computer Networks, vol. 43, pp. 499-518, 2003.

[98] R. Nagpal, H. Shrobe, and J. Bachrach, "Organizing a global coordinate system from local information on an ad hoc sensor network," in Proc. Sensor Networks 2nd International Workshop Inform, 2003.

[99] S. Capkun, M. Hamdi, and J. Hubaux, "GPS-free positioning in mobile ad-hoc networks," in 34th Hawaii International Conference on System Sciences, 2001, pp. 3481-3490.

[100] C. KWOK, D. FOX, and M. MEILA, "Real-time particle filters," Proceedings of the IEEE, vol. 92, no. 3, pp. 469-484, 2004

[101] V. Fox, J. Hightower, L. Lin, D. Schulz, and G. Borriello, "Bayesian filtering for location estimation," IEEE Pervasive Computing, vol. 2, no. 3, pp. 24-33, 2003.

[102] A. Ihler, I. Fisher, J.W., R. Moses, and A. Willsky, "Nonparametric belief propagation for self-localization of sensor networks," IEEE Journal on Selected Areas in Communications, vol. 23, no. 4, pp. 809819,2005

[103] J. Chen, K. Yao, and R. Hudson, "Source localization and beamforming," IEEE Signal Processing Magazine, vol. 19, no. 2, pp. 30-39, 2002.

[104] M. Rabbat and R. Nowak, "Distributed optimization in sensor networks," in Third International Symposium on Information Processing in Sensor Networks, 2004, pp. 20-27.

[105] T. Eren, D. Goldenberg, W. Whiteley, R. Yang, A. Morse, B. Anderson, and P. Belhumeur, "Rigidity and randomness in network localization," in IEEE INFOCOM, vol. 4, 2004, pp. 2673-2684.

[106] J. Aspnes, T. Eren, D. Goldenberg, A. Morse, W. Whiteley, Y. Yang, B. Anderson, and P. Belhumeur, "A theory of network localization," to appear in IEEE Transactions on Mobile Computing, 2006.

[107] D. Moore, J. Leonard, D. Rus, and S. Teller, "Robust distributed network localization with noisy range measurements," in the 2nd ACM Conf. on Embedded Networked Sensor Systems (SenSys'04), 2004, pp. $50-61$.

[108] B. Anderson, P. Belhumeur, T. Eren, D. Goldenberg, A. Morse, W. Whiteley, and R. Yang, "Graphical properties of easily localizable sensor networks," submitted for publication in Wireless Networks, 2005.

[109] R. Connelly, "Generic global rigidity," Discrete and Computational Geometry, vol. 33, pp. 549-563, 2005.

[110] B. Hendrickson, "Conditions for unique graph realizations," SIAM J. Comput., vol. 21, no. 1, pp. 65-84, 1992.

[111] D. Goldenberg, A. Krishnamurthy, W. MAness, R. Yang, A. Young, A. Morse, A. Savvides, and B. Anderson, "Network localization in partially localizable networks," in IEEE INFOCOM, vol. 1, 2005, pp. $313-326$.

[112] D. Niculescu and B. Nath, "Error characteristics of ad hoc positioning systems (APS)," in The 5th ACM international symposium on Mobile ad hoc networking and computing, 2004, pp. $20-30$.

[113] A. Savvides, W. L. Garber, R. L. Moses, and M. B. Srivastava, "An analysis of error inducing parameters in multihop sensor node localization," IEEE Transactions on Mobile Computing, vol. 4, no. 6, pp. $567-577,2005$.

[114] D. K. Goldenberg, P. Bihler, M. Cao, J. Fang, B. D. O. Anderson, A. S. Morse, and Y. R. Yang, "Localization in sparse networks using sweeps," in Proceedings of ACM MOBICOM, 2006, pp. 110 - 121. 
Guoqiang Mao received the Bachelor degree in electrical engineering, Master degree in engineering and $\mathrm{PhD}$ in telecommunications engineering in 1995, 1998 and 2002 respectively. After graduation from $\mathrm{PhD}$, he worked in "Intelligent Pixel Incorporation" as a Senior Research Engineer for one year. $\mathrm{He}$ joined the School of Electrical and Information Engineering, the University of Sydney in December 2002 where he is a Senior Lecturer now. His research interests include wireless sensor networks, wireless localization techniques, network QoS, telecommunications traffic measurement, analysis and modeling, and network performance analysis.

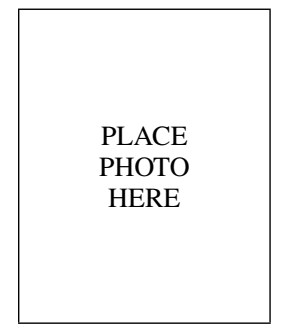

Barıs Fidan received the B.S. degrees in electrical engineering and mathematics from Middle East Technical University, Turkey in 1996, the M.S. degree in electrical engineering from Bilkent University, Turkey in 1998, and the Ph.D. degree in Electrical Engineering-Systems at the University of Southern California, Los Angeles, USA in 2003. After working as a postdoctoral research fellow at the University of Southern California for one year, he joined the Systems Engineering and Complex Systems Program of National ICT Australia and the Research School of Information Sciences and Engineering of the Australian National University, Canberra, Australia in 2005, where he is currently a researcher. His research interests include autonomous formations, sensor networks, adaptive and nonlinear control, switching and hybrid systems, mechatronics, and various control applications including high performance and hypersonic flight control, semiconductor manufacturing process control, and disk-drive servo systems.

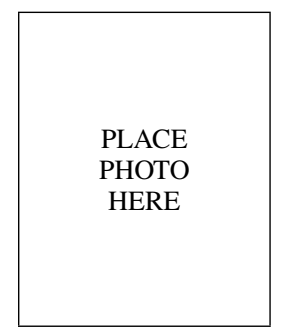

Brian D. O. Anderson was born in Sydney, Australia, and received his undergraduate education at the University of Sydney, with majors in pure mathematics and electrical engineering. He subsequently obtained a $\mathrm{PhD}$ degree in electrical engineering from Stanford University. Following completion of his education, he worked in industry in Silicon Valley and served as a faculty member in the Department of Electrical Engineering at Stanford. He was Professor of Electrical Engineering at the University of Newcastle, Australia from 1967 until 1981 and is now a Distinguished Professor at the Australian National University and Chief Scientist of National ICT Australia Ltd. His interests are in control and signal processing. He is a Fellow of the IEEE, Royal Society London, Australian Academy of Science, Australian Academy of Technological Sciences and Engineering, Honorary Fellow of the Institution of Engineers, Australia, and Foreign Associate of the US National Academy of Engineering. $\mathrm{He}$ holds doctorates (honoris causa) from the Université Catholique de Louvain, Belgium, Swiss Federal Institute of Technology, Zürich, Universities of Sydney, Melbourne, New South Wales and Newcastle. He served a term as President of the International Federation of Automatic Control from 1990 to 1993 and as President of the Australian Academy of Science between 1998 and 2002. His awards include the IEEE Control Systems Award of 1997, the 2001 IEEE James H Mulligan, Jr Education Medal, and the Guillemin-Cauer Award, IEEE Circuits and Systems Society in 1992 and 2001, the Bode Prize of the IEEE Control System Society in 1992 and the Senior Prize of the IEEE Transactions on Acoustics, Speech and Signal Processing in 1986. 